\title{
Ferulic Acid Produced by Lactobacillus fermentum NCIMB 5221 Reduces Symptoms of Metabolic Syndrome in Drosophila melanogaster
}

Susan Westfall', Nikita Lomis ${ }^{1}$, Surya Pratap Singh ${ }^{2}$ and Satya Prakash ${ }^{1 *}$

${ }^{1}$ Department of Biomedical Engineering, Department of Experimental Medicine, Faculty of Medicine, Biomedical Technology and Cell Therapy Research Laboratory, McGill University, 3775 University Street, Montreal, Quebec, H3A2B4, Canada

${ }^{2}$ Department of Biochemistry, Faculty of Science, Banaras Hindu University, Varanasi, Uttar Pradesh, India

\begin{abstract}
The gut microbiota is an intricate community of thousands of bacterial species living in the gastrointestinal tract critical for vitamin synthesis, mineral absorption, digestion of otherwise indigestible fibers and the extraction of energy from food. Recently, the health of the gut microbiota's community architecture has become linked to energy-regulatory diseases including metabolic syndrome: a collection of symptoms including hyperglycemia, hypercholesterolemia, high blood pressure, increased abdominal fat and triglyceride levels. However, the mechanism of communication between the gut microbiota and the host energy metabolism remains elusive. The current study shows that ferulic acid (FA) produced by the intrinsic ferulic acid esterase activity of the probiotic bacteria Lactobacillus fermentum NCIMB 5221 (Lf5221) can dose-dependently rescue the phenotypic markers of diet-induced diabetes and obesity in Drosophila melanogaster. In Drosophila exposed to either a high-sugar or high-fat diet, living Lf5221 at 2.5 or $7.5 \times 10^{9} \mathrm{CFU} / \mathrm{ml}$ media effectively rescued whole-body weight, glucose, trehalose and triglyceride levels. All of the aforementioned effects were lost in heat-inactivated bacteria indicating that a metabolic product is responsible. Likewise, FA at $0.5 \mathrm{mM}$ in the metabolically challenged Drosophila models reared similar effects on the physiological markers while also reducing hyperglycemia in the circulating hemolymph. On the signaling level, the high-sugar diet predictably had an elevated expression of the Drosophila insulin-like peptides 2, 3 and 5 and in the high-fat diet, an increase in fatty acid synthase, acetyl-CoA carboxylase and phosphoenolpyruvate carboxykinase expression. On both diets, Lf5221 and FA rescued gene expression, at different concentrations, to the level of controls. Examining the mechanistic gene expression, both Lf5221 and FA rescued expression of dFOXO and dTOR, but not dAkt indicating that FA produced by Lf5221 is acting on one of the downstream-signaling molecules from the insulin receptor, possibly dTOR: an overall energy regulator in flies and humans alike. The present study for the first time outlines a streamlined mechanism for how the gut microbiota communicates with the host's energy-regulating metabolism. Proper supplementation with ferulic acid esterase active probiotics such as Lf5221 could potentially prevent or alleviate symptoms of metabolic syndrome and other energy-regulating diseases including diabetes, obesity and neurodegeneration.
\end{abstract}

Keywords: Metabolic syndrome; Probiotics; Lactobacillus fermentum; Ferulic acid; Diabetes; Obesity; TOR

\begin{abstract}
Abbreviations
ACC: Acetyl-CoA Carboxylase; CFU:Colony Forming Units; dAkt: Drosophila Protein Kinase B; dilp: Drosophila insulin-like peptide; dFOXO: Drosophila Forkhead Box Protein O; dTOR: Drosophila Target of Rapamycin; FA: Ferulic Acid; FAE: Ferulic Acid Esterase; FAS: Fatty Acid Synthase; GIT: Gastrointestinal Tract; HFD: High Fat Diet; HSD: High Sugar Diet; IlS: Insulin-like Signaling; Lf5221: Lactobacillus fermentum NCIMB 5221; MRS: Man-Rogosa-Sharpe; PEPCK: Phosphoenolpyruvate Carboxykinase; Upd2: Unpaired 2
\end{abstract}

\section{Introduction}

The gut microbiota is constituted by a plethora of bacterial species residing symbiotically in the gastrointestinal tract (GIT). These estimated $10^{13}-10^{14}$ bacterial cells are indispensable to the host's wellbeing as they form a dense synergistic ecosystem that impacts host digestion of vitamins, minerals and otherwise indigestible fibers, extraction of energy from foods, production of signaling molecules and hormones and modulation of gastrointestinal dynamics including motility and transit time [1]. The microbiota may be considered as an organ onto itself, being responsible for a variety of physiological activities including metabolism, neurological development, energy homeostasis, immune regulation and fundamental signaling cascades.

Recently, the health of the gut microbiota has been linked to the pathology of metabolic syndrome, a growing health and economic concern. Metabolic syndrome is a collection of energy-regulating risk factors including hyperglycemia, pre-diabetes, abdominal obesity, elevated cholesterol and triglycerides and high blood pressure leading to an elevated risk of cardiovascular disease [2]. In the United States alone, there was an increased prevalence from 32.9 to $34.7 \%$ diagnosed cases, mostly in women and persons over the age of 60 years [3]. Although risk factors vary based on demographic and gender differences [4], the increasing global sedentary lifestyle and proportionally aging population has made the incidence of metabolic syndrome rise to epidemic proportions [5].

It has been recently recognized that the gut microbiota is pinnacle for regulating metabolic energy homeostasis, especially in the context of metabolic syndrome [6,7]. Depending on an individual's diet, the gut

*Corresponding author: Satya Prakash, Department of Biomedical Engineering Department of Experimental Medicine, Faculty of Medicine, Biomedical Technology and Cell Therapy Research Laboratory, McGill University, 3775 University Street, Montreal, Quebec, H3A2B4, Canada, Tel: 1-514-398-3676; E-mail: satya.prakash@mcgill.ca

Received June 05, 2016; Accepted June 30, 2016; Published July 08, 2016

Citation: Westfall S, Lomis N, Singh SP, Prakash S (2016) Ferulic Acid Produced by Lactobacillus fermentum NCIMB 5221 Reduces Symptoms of Metabolic Syndrome in Drosophila melanogaster. J Microb Biochem Technol 8: 272-284. doi: 10.4172/1948-5948.1000297

Copyright: ( 2016 Westfall S, et al. This is an open-access article distributed under the terms of the Creative Commons Attribution License, which permits unrestricted use, distribution, and reproduction in any medium, provided the original author and source are credited. 
microbiota will adapt to the nutritional phenotype and be composed of different proportions of beneficial and pathogenic species. For example, on a typical western diet laden with refined sugar and fats, there will be an over representation of the phyla Firmicutes and an under representation of the phyla Bacteriodetes. An increase in this ratio is linked to adiposity, hypertriglyceridemia, diabetes and increased lipopolysaccharide (originating from gram-negative bacteria) levels that contribute to systemic inflammation [8].

The role of gut microbiota in metabolic syndrome was first demonstrated by Backhed et al. using germ-free rodents, animals free of all microorganisms both in and on them. When the microbiota of conventionally raised animals was transferred to germ-free rodents, the latter demonstrated a $60 \%$ increase in adiposity and insulin resistance, regardless of decreased muscle mass and food intake [9]. Similarly, Ley et al. demonstrated that ob/ob mice, a genetic model of obesity due to a mutation in the leptin gene, have a distinct microbiota when compared to their lean counterparts. Specifically, the obese mice demonstrated a $50 \%$ reduction in Bacteroidetes (Gram-negative) and a 50\% increase in Firmicutes (Gram-positive) counts [10]. Interestingly, in a very recent study, it was shown using the gut microbiota from mouse twins discordant for obesity, that there is a strong interaction between the microbiota and diet [11]

Certain probiotic bacterial strains have demonstrated important anti-inflammatory potential, both in vitro $[12,13]$ and in vivo [14], which is beneficial for the management of chronic systemic low-grade inflammation present in metabolic syndrome. Probiotic strains have also demonstrated the capability to lower cholesterol levels, linked to atherogenic dyslipidemia [15]. In addition, specific probiotic strains have been shown to impact the development of obesity in humans [16]. In diabetes, there is a characteristic reduction of Firmicutes and other bacterial species including, Bacteroides vulgatus, Faecalibacterium prauznitzii and the Bifidobacterium, Lactobacillus and Roseburia genus [3]. This creates an un-healthy GIT environment that is more prone to the growth of opportunistic pathogens and pro-inflammatory species that aggravate metabolic phenotypes. In addition, an increase in fecal levels of Lactobacillus gasseri, Streptococcus mutans and Escherichia coli is predictive of insulin resistance. When insulin-resistant males received lean donor fecal transplantations, there was a significant increase in intestinal microbial diversity and a distinct increase in butyrateproducing bacteria such as Roseburia and Faecalibcaterium spp. in the feces and Eubacterium halii in the small intestine which improves GIT health and alleviates the metabolic symptoms.

With the microbiota's importance in health and disease, it has become a therapeutic target [17]. There are several ways that the gut microbiota can be altered including the consumption of healthpromoting bacterial probiotic species, indigestible fibers or prebiotics and a combination of the two known as synbiotics. The Food and Agriculture Organization of the United Nations and the WHO define probiotic bacteria as "live microorganisms which, when administered in adequate amounts, confer a health benefit on the host". Several probiotics have been identified to be beneficial to the metabolic syndrome due to their insulin-regulating, anti-obesity and antiinflammatory effects. Many probiotic species secrete metabolically active products or digest otherwise indigestible fibers (prebiotics) to produce signaling molecules with downstream metabolically active effects. These include the short-chain fatty acids (SCFAs), that regulate energy metabolism (reviewed in [18]), but there are many other products that also elicit beneficial effects.

In the present study, the ferulic acid esterase (FAE)-active probiotic L. fermentum NCIMB 5221 (herein referred to as Lf5221) reduced a variety of metabolic stress markers induced by a highsugar or a high-fat diet in Drosophila melanogaster. Ferulic acid (FA, 4-hydroxy-3-methoxy cinnamic acid) is released from plant polymers, mostly lignans and other esterified substances by the FAE activity of bacteria [19]. FA boasts many beneficial properties including reducing insulin resistance, triglycerides, cholesterol blood pressure, chronic inflammation, oxidative stress and more physiological manifestations of metabolic distress (rev. in [20]. It is in this context that the specific physiological and molecular effects of Lf5221-generated FA on metabolically challenged Drosophila melanogaster was investigated in order to delineate a molecular mechanism of communication from probiotic therapy and changes in the gut microbiota with metabolic management of host physiology.

\section{Materials and Methods}

\section{Bacterial strain and culture conditions}

L. fermentum NCIMB 5221 (Lf5221) was obtained from the NCIMB Culture Collection (Aberdeen, Scotland, UK). Cells were cultured with Man-Rogosa-Sharpe (MRS) media obtained from Sigma Aldrich (Oakville, ON, Canada) at $37^{\circ} \mathrm{C}$ on MRS-agar plates or in liquid media. After one round of liquid culture, several bacterial stocks were made in MRS containing 20\% $(v / v)$ glycerol and stored at $-80^{\circ} \mathrm{C}$. As constant culturing was required to carry out all experiments, bacterial stocks were renewed from the frozen stock bi-weekly in order to maintain culture purity. To preform each individual experiment, a $1 \%(v / v)$ inoculum was used for subculturing, incubated at $37^{\circ} \mathrm{C}$ for $18 \mathrm{~h}$ and removed immediately before use. To prepare the individual Drosophila bottles, the overnight culture was centrifuged at $4000 \mathrm{rpm}$ for $10 \mathrm{~min}$ at $4^{\circ} \mathrm{C}$. The pellet was washed once and resuspended in $0.85 \%(w / v)$ physiological saline. Total colony counts were determined by spectrophotometry compared to a standard curve prepared with colony forming units (CFUs) on MRS plates. Heat inactivated bacteria were prepared by heating the concentrated bacterial solution at $95^{\circ} \mathrm{C}$ for $20 \mathrm{~min}$. This method was verified by plating heat-treated bacterial cells on MRS-agar plates at various dilutions and counting any possible CFUs. With this treatment regime, there were no CFUs at any dilution after $72 \mathrm{~h}$ of incubation at $37^{\circ} \mathrm{C}$.

\section{Drosophila melanagaster strains and media}

Wild type Drosophila melanogaster (Oregon R) were procured from the Bloomington Drosophila Stock Center (Indiana University, Bloomington Indiana). Flies were reared on a standard cornmealsucrose-yeast media without active yeast culture prepared by boiling the cornmeal $(83 \mathrm{~g})$, sucrose $(50 \mathrm{~g})$ and yeast extract $(30 \mathrm{~g})$ in distilled water for $30 \mathrm{~min}$. Drosophila were kept in controlled conditions with a $12 \mathrm{~h}: 12 \mathrm{~h}$ light-dark cycle at $25^{\circ} \mathrm{C}$. Baseline media contained $0.15 \mathrm{M}$ sucrose whereas the high-sugar diet (HSD) contained 1.0 M sucrose and the high-fat diet (HFD) contained an additional 20\% $(v / v)$ coconut oil. Diabetes or obesity was induced by transferring 7 day old flies reared and kept on normal probiotic-containing media to a HSD or HFD, respectively also containing the probiotic for 21 days at which point the flies were sampled. Inoculated media was prepared by partitioning the concentrated bacterial culture into the cooled, yet liquid, media to final concentrations of $5.0 \times 10^{8}$ to $7.5 \times 10^{9} \mathrm{CFU} / \mathrm{ml}$ media. This is a verified method of oral-inoculation to flies and it was verified that bacterial cells remained viable in the Drosophila media for up to two weeks before a detectable loss of concentration by daily CFU counting. Nevertheless, flies were transferred to new inoculated bottles every 3-4 days during the course of an experiment. 
Citation: Westfall S, Lomis N, Singh SP, Prakash S (2016) Ferulic Acid Produced by Lactobacillus fermentum NCIMB 5221 Reduces Symptoms of Metabolic Syndrome in Drosophila melanogaster. J Microb Biochem Technol 8: 272-284. doi: 10.4172/1948-5948.1000297

\section{Drosophila flies sampling}

After three weeks of treatment with Lf5221, FA and/or HSD/HFD flies were anesthetized in an ether chamber at $10 \mathrm{am}, 2 \mathrm{~h}$ after lightson. Hemolymph was collected by piercing the Drosophila's thorax with a thin needle and collecting 40 flies in a small tube with several holes situated in a larger centrifuge tube. Samples were centrifuged at 5000 $\mathrm{rpm}$ for $5 \mathrm{~min}$ at $4^{\circ} \mathrm{C}$ to collect hemolymph. Samples were immediately placed on ice and all subsequent experiments were conducted immediately after collection. Whole-body homogenates were prepared by homogenizing on ice 20 flies in $200 \mu \mathrm{l}$ of $10 \mathrm{mM}$ Tris-EDTA, $0.5 \%$ Triton X-100 buffer, $\mathrm{pH}$ 7.4. Samples were gently centrifuged for $3 \mathrm{~min}$ at $1000 \mathrm{rpm}$ and $4^{\circ} \mathrm{C}$ to remove debris.

\section{Measure of self-reproducibility}

To measure the self-reproducibility of Lf5221 in Drosophila after oral inoculation, two-day-old flies were starved in empty vials for $3 \mathrm{~h}$ (to enable synchronous feeding) prior to being transferred to fresh vials containing various concentrations of Lf5221. After $24 \mathrm{~h}$ of inoculation, flies were surface sterilized in $10 \%$ bleach, rinsed thrice in distilled water and transferred to sterile (autoclaved) media in a sterile hood. The removal of all surface bacteria and feces was verified by plating the wash water on MRS-agar plates to ensure no CFUs formed within 72 $h$. The surface sterilization and transfer to sterile media was repeated daily to limit cross-contamination of feces to flies. Samples of flies were taken daily and assessed for the presence of Lf5221 by the extraction of bacterial DNA with the All-4-One DNA extraction kit with the added step of lysozyme digestion (BIO BASIC Int., Markham, Canada) followed by real-time PCR with species-specific primers.

\section{Metabolic marker determination: Weight, glucose, trehalose, triglycerides}

Body weight was assessed by weighing ten flies in replicates of five. Glucose and trehalose (the glucose storage molecule in Drosophila) measurements were taken from both hemolymph and whole-body homogenates of Drosophila. For the whole-body homogenates, the total protein content was determined using a Bradford Assay and resultant quantification of metabolic markers was standardized against the total protein content in order to account for variations in fly mass. Following, the homogenate was heat-treated for $20 \mathrm{~min}$ at $70^{\circ} \mathrm{C}$ to remove any complexes. Glucose levels were measured in $2 \mu \mathrm{l}$ of hemolymph or $5 \mu$ of whole-body homogenate using the Glucose (HK) Assay kit (Sigma, Oakvilla, ON, Canada) according to the manufacturer's instructions. Whole-body trehalose was determine by digesting $2 \mu \mathrm{l}$ of hemolymph or $5 \mu \mathrm{l}$ of whole-body homogenate with $0.4 \mu \mathrm{l}$ or $1 \mu \mathrm{l}$ of trehalase (Sigma, Oakville, ON, Canada), respectively at $37^{\circ} \mathrm{C}$ for $4 \mathrm{~h}$. After incubation, total glucose levels were measured with the Glucose (HK) Assay kit and the total amount of digested trehalose was determined by subtracting the values for total glucose. Wholebody triglycerides were determined in $10 \mu \mathrm{l}$ of homogenate using the Triglycerides Liquicolor Test Mono (Stanbio, TX, USA) according to the manufacteur's instructions.

\section{RNA isolation and real-time PCR}

RNA was extracted from whole flies using Trizol (ThermoFisher, MA, USA) according to the manufacturer's instructions. cDNA was synthesized from $1 \mu \mathrm{g}$ of RNA measured with the ND-2000 Nanodrop (FisherScientific, Ottawa, ON, Canada) using the High-Capacity cDNA Synthesis Kit (ThermoFisher, MA, USA) according to the manufacturer's instructions. Real-time PCR was conducted on the Eco Real-time PCR System (Illumina, CA, USA) using SybrGreen detection method (Diamed, Mississauga, ON, Canada). Primer pairs and annealing temperatures are listed in Table 1.

\section{Statistics}

All experiments were conducted in 3 independent trials with $n=5$ replicates in each experiment. Variations in body weight and motility over the dose-curves of probiotic were assessed via one-way ANOVA analyses with Tukey post-hoc tests. Significance of metabolic markers and genetic expression was assessed with two-way ANOVA with

\begin{tabular}{|c|c|c|c|}
\hline Gene Name & Sequence & Annealing Temperature & References \\
\hline \multirow{2}{*}{ Dilp 2} & forward: 3'- AGCAAGCCTTTGTCCTTCATCTC - 5' & \multirow{2}{*}{$50^{\circ} \mathrm{C}$} & \multirow{2}{*}{ [21] } \\
\hline & reverse: 3'- ACACCATACTCAGCACCTCGTTG - 5' & & \\
\hline \multirow{2}{*}{ Dilp 3} & forward: 3'- TGTGTGTATGGCTTCAACGCAATG - 5' & \multirow{2}{*}{$50^{\circ} \mathrm{C}$} & \multirow{2}{*}{ [21] } \\
\hline & reverse: 3'- CACTCAACAGTCTTTCCAGCAGGG - 5' & & \\
\hline \multirow{2}{*}{ Dilp 5} & forward: 3'- GAGGCACCTTGGGCCTATTC - 5' & \multirow{2}{*}{$57^{\circ} \mathrm{C}$} & \multirow{2}{*}{ [21] } \\
\hline & reverse: 3'- CATGTGGTGAGATTCGGAGC - 5' & & \\
\hline \multirow{2}{*}{$A C C$} & forward: 3'- TTAGTCAGCTGCAGGCAAAGG - 5' & \multirow{2}{*}{$54^{\circ} \mathrm{C}$} & \multirow{2}{*}{ [22] } \\
\hline & reverse: 3'- CGGAAGCTAACGCCACACA - 5' & & \\
\hline \multirow{2}{*}{ FAS } & forward: 3' - CAACAAGCCGAACCCAGATCTT - 5' & \multirow{2}{*}{$50^{\circ} \mathrm{C}$} & \multirow{2}{*}{ [22] } \\
\hline & reverse: 3'- CAAAGGAGTTCAGGCCGATGAT - 5' & & \\
\hline \multirow{2}{*}{ PEPCK } & forward: 3'- CGCCCAGCGACATGGATGCT - 5' & \multirow{2}{*}{$60^{\circ} \mathrm{C}$} & \multirow{2}{*}{ [22] } \\
\hline & reverse: 3'- GTACATGGTGCGACCCTTCA - 5' & & \\
\hline \multirow{2}{*}{$d T O R$} & forward: 3'- GGCCGTCCAGGTTCAAAAAC - 5' & \multirow{2}{*}{$59^{\circ} \mathrm{C}$} & \multirow{2}{*}{ This study } \\
\hline & reverse: 3'-AATCCGGCGATAGTTCCGTC - 5' & & \\
\hline \multirow{2}{*}{$d A k t$} & forward: 3'- GAGTCGTGTGCTCAAGTCCA - 5' & \multirow{2}{*}{$59^{\circ} \mathrm{C}$} & \multirow{2}{*}{ This study } \\
\hline & reverse: 3'- TGCATCACAAAACACAGGCG - 5' & & \\
\hline \multirow{2}{*}{$d F O X O$} & forward: 3'- TCGCCGAACTCAGTAACCAC - 5' & \multirow{2}{*}{$59^{\circ} \mathrm{C}$} & \multirow{2}{*}{ This study } \\
\hline & reverse: 3'- TCCTATCAAAGTAGAGGCGCA - 5' & & \\
\hline \multirow{2}{*}{ Rp49 } & forward: 3'- AGATCGTGAAGAAGCGCACCAAG - 5 ' & \multirow{2}{*}{$52^{\circ} \mathrm{C}$} & \multirow{2}{*}{ [23] } \\
\hline & reverse: 3'- CACCAGGAACTTCTTGAATCCGG - 5' & & \\
\hline \multirow{2}{*}{ Lf5221 } & forward: 3' - AGTCACGGCTAACTACGTGC- 5' & \multirow{2}{*}{$59^{\circ} \mathrm{C}$} & \multirow{2}{*}{ This study } \\
\hline & reverse: 3' - TCTACGCATTCCACCGCTAC- 5' & & \\
\hline
\end{tabular}

Table 1: Primer pairs and annealing temperatures. 
Tukey post-hoc analyses. Significance was determined if $p<0.05$ and evaluation at $p<0.01$.

\section{Results}

Orally administered Lactobacillus fermentum self-replicates in the Drosophila colon

L. fermentum NCIMB 5221 (Lf5221) is not an endogenous bacterial species in Drosophila although other Lactobacillus species including $L$. plantarum and L. brevis are. To determine if Lf5221 can self-replicate in the Drosophila colon, flies were inoculated with various concentrations of Lf5221 for $24 \mathrm{~h}$ and then transferred daily to new sterile media. Using real-time PCR, a baseline of Lf5221 was observed in the control group, likely indicating some non-specificity in the primer pairs for the commensal Lactobacillus species. Nevertheless, there was a dosedependent increase in Lf5221 present in the colon dependent on the orally inoculated dose and these concentrations either remained the same $\left(5.0 \times 10^{8} \mathrm{CFU} / \mathrm{ml}\right)$ or increased slightly over time for the 2.5 $\times 10^{9} \mathrm{CFU} / \mathrm{ml}(F(1,9)=5.34, p<0.05)$ and the $7.5 \times 10^{9} \mathrm{CFU} / \mathrm{ml}$ group $(F(1,9)=6.43, p<0.05)$, (Figure 1). Since there was an apparent increase in bacterial species over time without exogenous supply, it can be concluded that Lf5221 can self-replicate within the conditions of the Drosophila colon.

\section{L. fermentum rescues physiological markers of diabetes in Drosophila fed high-sugar diet}

High body weight is indicative of dysregulated energy metabolism and a state often materializing with diabetes. Flies fed on a highsugar diet (HSD; $1.0 \mathrm{M}$ sucrose) experienced a $26 \%$ increase in body weight $(p<0.01)$ compared to flies fed a normal diet $(0.15 \mathrm{M}$ sucrose). However, co-feeding flies with Lf5221 rescued the weight gain in a dose-dependent manner such that at the highest tested concentration $\left(7.5 \times 10^{9} \mathrm{CFU} / \mathrm{ml}\right)$ there was no difference in the average weight of flies fed a HSD compated to controls ( $p>0.05$; Figure 2a). Flies fed on a HSD supplemented with heat-inactivated Lf5221 did not have a reduction in weight gain concluding that the metabolic activity of $L$. fermentum is necessary for the weight preservation effects.
Circulating levels of glucose (Figure $2 \mathrm{~b}$ ) and trehalose (Figure 2c) were similarly elevated in flies fed a HSD ( $>50 \%$ and $200 \%$, respectively; $p<0.01)$. However, neither living nor heat-inactivated Lf5221 significantly rescued circulating glucose or trehalose levels (Figures $2 \mathrm{~b}$ and $2 c ; p>0.05)$.

Whole body glucose and trehalose are indicative of the physiological storage of glucose. Flies fed on a HSD experienced elevated whole-body glucose $(>45 \%$; Figure $2 \mathrm{~d}, p<0.01)$ and wholebody trehalose levels $(>33 \%$; Figure $2 \mathrm{e}, p<0.01)$. Co-treatment with $5.0 \times 10^{8} \mathrm{CFU} / \mathrm{ml}$ Lf5221 reduced the amount of whole-body glucose by $25 \%$ from HSD-treated flies (Figure $2 \mathrm{~d} ; p<0.01$ ) whereas at 7.5 $\times 10^{9} \mathrm{CFU} / \mathrm{ml}$, trending towards the same level as controls (Figure $2 \mathrm{~d}, p=0.06$ ). The main storage carbohydrate in flies is trehalose (comparable to glycogen in humans) and the elevated trehalose levels in response to the HSD was dose-dependently reduced by Lf5221 to a level comparable to controls at $5.0 \times 10^{8} \mathrm{CFU} / \mathrm{ml}$ (Figure 2e; $p<0.05$ ), while heat-inactivated Lf5221 did not affect whole-body trehalose levels.

Finally, whole-body triglycerides are also typically elevated in animals fed a HSD due to the broad dysregulation of energy metabolism. Likewise, flies fed on a HSD experienced almost a $50 \%$ increase in body weight (Figure 2f; $p<0.01$ ) with respect to controls. In a dose-dependent manner, Lf5221 reduced the total triglyceride concentrations, where at $7.5 \times 10^{9} \mathrm{CFU} / \mathrm{ml}$ reduced triglyceride concentrations to the level of control (Figure 2f; $p>0.05$ ).

\section{Ferulic acid produced by $L$. fermentum NCIMB 5221 rescues diabetic markers in Drosophila fed a high sugar diet}

Flies were reared on media containing various concentrations of FA and remained on the normal media containing FA for 7 days before being transferred to the HSD, also supplemented with FA. After three weeks, the same parameters as outlined for Lf5221 were measured. Flies grown on a HSD showed a $32 \%$ increase in body weight, which was reduced when flies were reared on media containing $0.1 \mathrm{mM} F A$ (Figure 3a; $p<0.01$ ). In contrast to Lf5221, the elevation in circulating

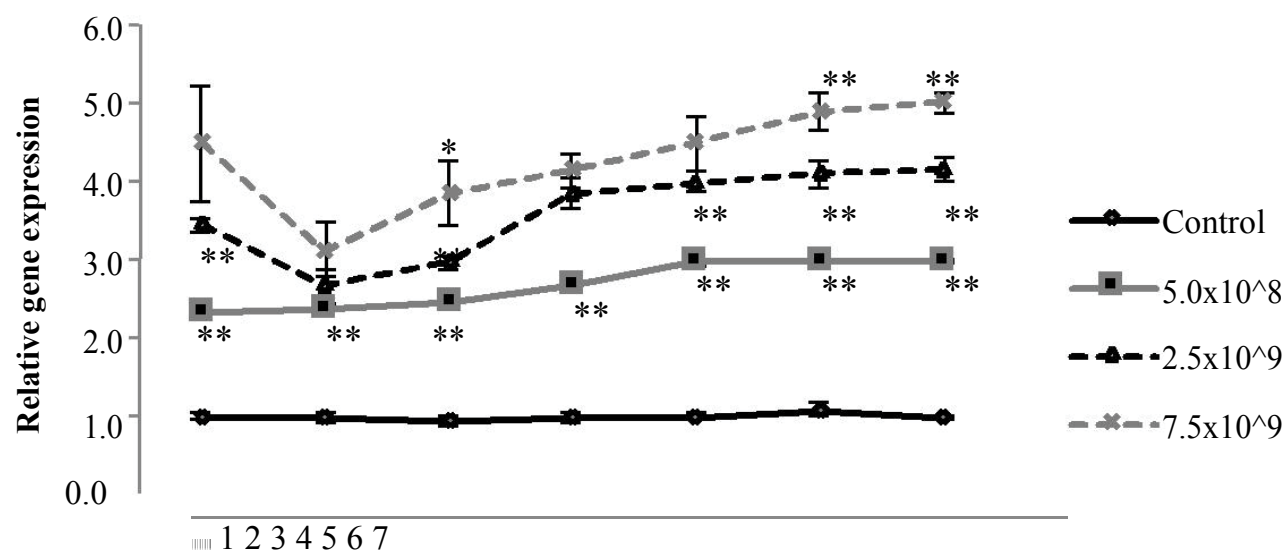

Time (Days)

Figure 1: L. fermentum NCIMB 5221 persistence in the Drosophila colon after oral infection.

Flies were orally infected with four concentrations of $L$. fermentum NCIMB 5221 (CFU/ml) (Control - solid black lines with diamonds), $5.0 \times 10^{8} \mathrm{CFU} / \mathrm{ml}$ (grey lines with squares), $2.5 \times 10^{9} \mathrm{CFU} / \mathrm{ml}$ (black dotted lines with diamonds) and $7.5 \times 10^{9} \mathrm{CFU} / \mathrm{ml}$ (dotted grey lines with crosses) for $24 \mathrm{~h}$. Flies were transferred to sterile bottles daily and sampled. Total $L$. fermentum NCIMB 5221 counts were assessed with real-time PCR of species-specific primers. Each sample represents $n=5$ replicates and it represented as the average gene expression $+/$ - standard error. Error points ${ }^{*} p<0.05$ and ${ }^{* *} p<0.01$ represent the difference between the indicated dose point, and the point of the one-step lower concentration. 
glucose levels (almost doubled in HSD-treated flies) was significantly reduced by $50 \%$ at $0.5 \mathrm{mM}$ FA (Figure $3 \mathrm{~b} ; p<0.05$ ). The effect of FA was even more pronounced for circulating trehalose where FA completely rescued the elevation in trehalose at $0.1 \mathrm{mM}$ (Figure $3 \mathrm{c}$; $p<0.01)$. FA also had similar effects as Lf5221 for the whole-body glucose and trehalose levels. At $0.1 \mathrm{mM}$ and $0.5 \mathrm{mM}$, FA rescued the $45 \%$ HSD-mediated elevation in total glucose (Figure $3 \mathrm{~d} ; p<0.01$ ) and trehalose (Figure 3e; $p<0.05$ ) levels, respectively. Finally, it was further demonstrated that FA rescued the elevated total triglycerides levels in HSD-treated flies at $0.5 \mathrm{mM}$ (Figure 3f; $p<0.05$ ). Interestingly, it appears that $0.5 \mathrm{mM}$ is an optimal dose of FA and that above this amount $(1.0 \mathrm{mM})$ there is a less beneficial effect on the diabetic parameters of HSD-treated flies.

\section{Dilp expression is altered by ferulic acid producing $L$. fermentum NCIMB 5221 in Drosophila fed a high-sugar diet}

Drosophila insulin-like peptide (dilp) expression in Drosophila is modulated and regulated similarly to insulin signaling in mammals. Is it thus expected that dilp expression will increase in response to HSD equivalent to insulin-resistance in mammals. Indeed, an increase of approximately 2 -fold for both dilp 2 and 3 was noted in flies fed a HSD compared to controls (Figure 4; $p<0.05$ ), however there was an insignificant change in dilp5 (Figures $4 \mathrm{e}$ and $4 \mathrm{f} ; p>0.05$ ). With Lf5221 co-treatment, there was a complete rescue in dilp2 and 3 expression at the lowest Lf5221 dose $5.0 \times 10^{8} \mathrm{CFU} / \mathrm{ml}$ (Figures $4 \mathrm{a}$ and $4 \mathrm{c} ; p>0.05$ ) which was not apparent in heat-inactivated Lf5221-treated flies. FA
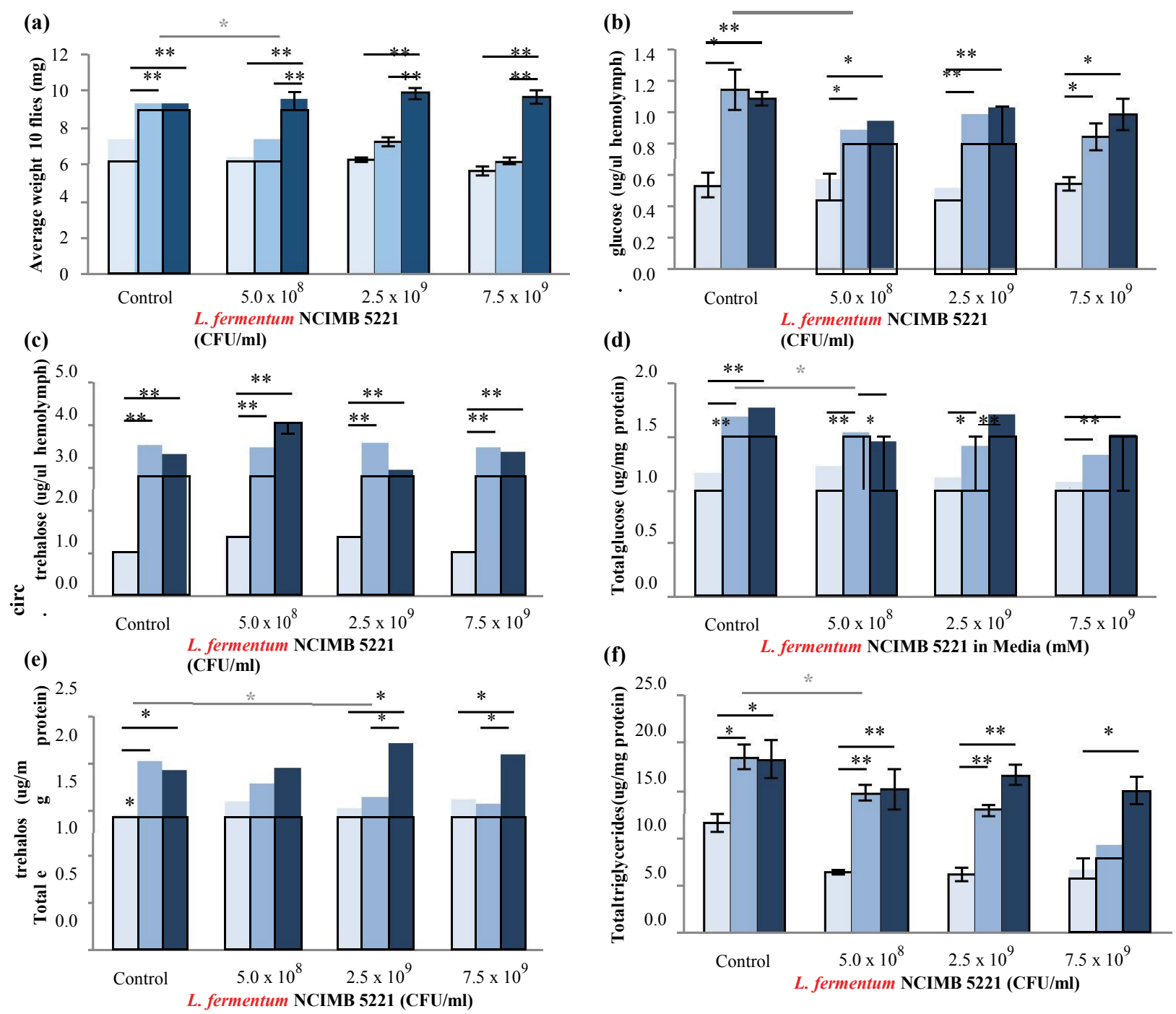

Figure 2: Metabolic markers of diet-induced diabetes are affected by oral treatment of the live probiotic $L$. fermentum NCIMB 5221 . Flies were reared on media containing various concentrations of Lf5221. After eclosion, flies remained on the probiotic-inoculated media for 7 days and were then subsequently transferred to the high-sugar media (HSD) containing Lf5221 for 21 days to induce diabetes together with the indicated probiotic. All markers were measured in control (light-blue bars), HSD with live Lf5221 (medium-blue bars) and HSD with heat-inactivated Lf5221 (dark-blue bars) treated groups at doses of 0 , $5.0 \times 10^{8}, 2.5 \times 10^{9}$ and $7.5 \times 10^{9} \mathrm{CFU} / \mathrm{ml}$. Various aspects of metabolic health were assessed including (a) average weight of 10 flies, (b) circulating levels of glucose normalized to I of hemolymph, (c) circulating levels of trehalose normalized to I of hemolymph, (d) whole-body glucose normalized to mg of protein, (e) whole body trehalose normalized to $\mathrm{mg}$ of protein and (f) whole body triglycerides also normalized to $\mathrm{mg}$ of protein. All values represent $n=5$ independent experiments $+/-$ the standard error. Significance is indicated as ${ }^{*} p<0.05$ or ${ }^{* *} p<0.01$ where black lines indicate difference between treatments groups and grey lines indicate the first significance difference between dose groups for the HSD supplemented with live Lf5221. 
(a)
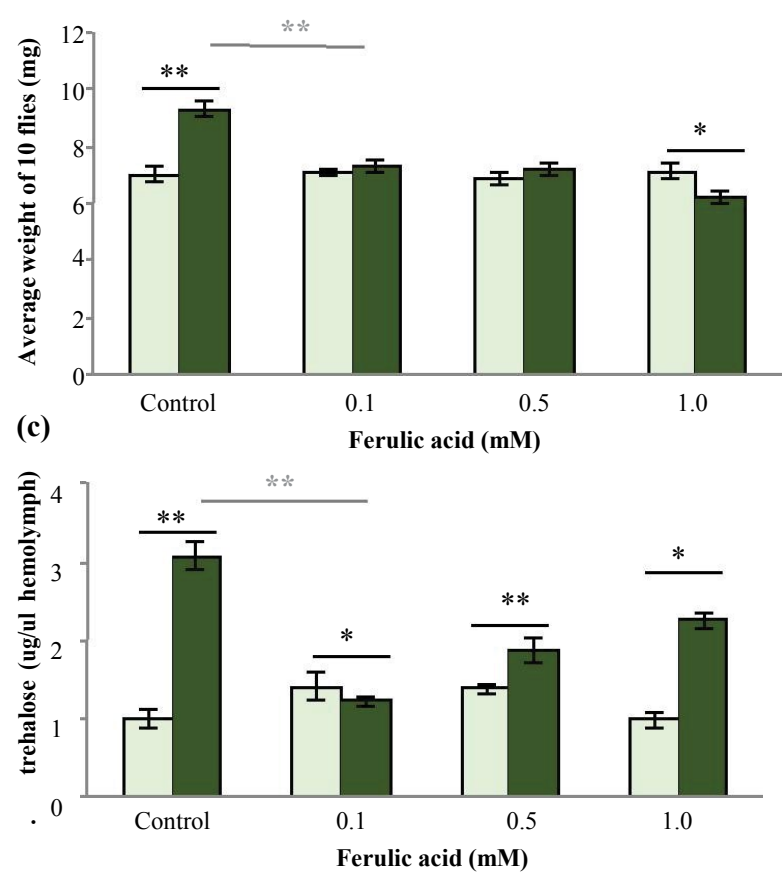

(e)

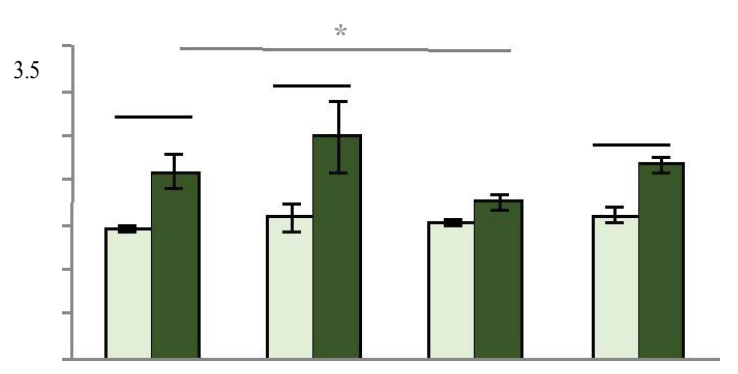

(b)

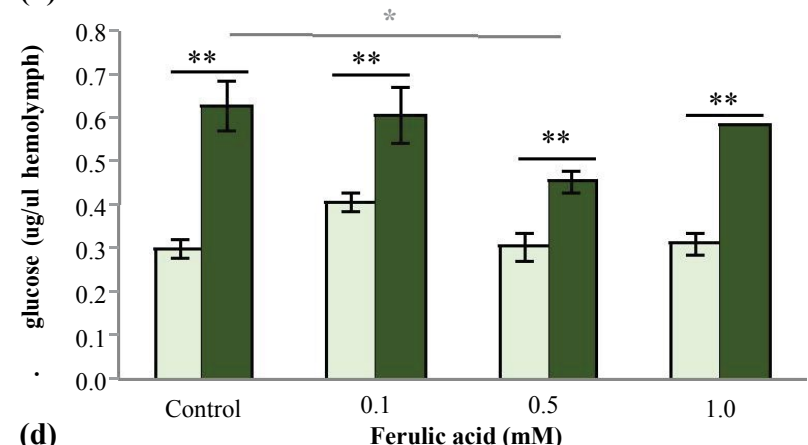

(d)
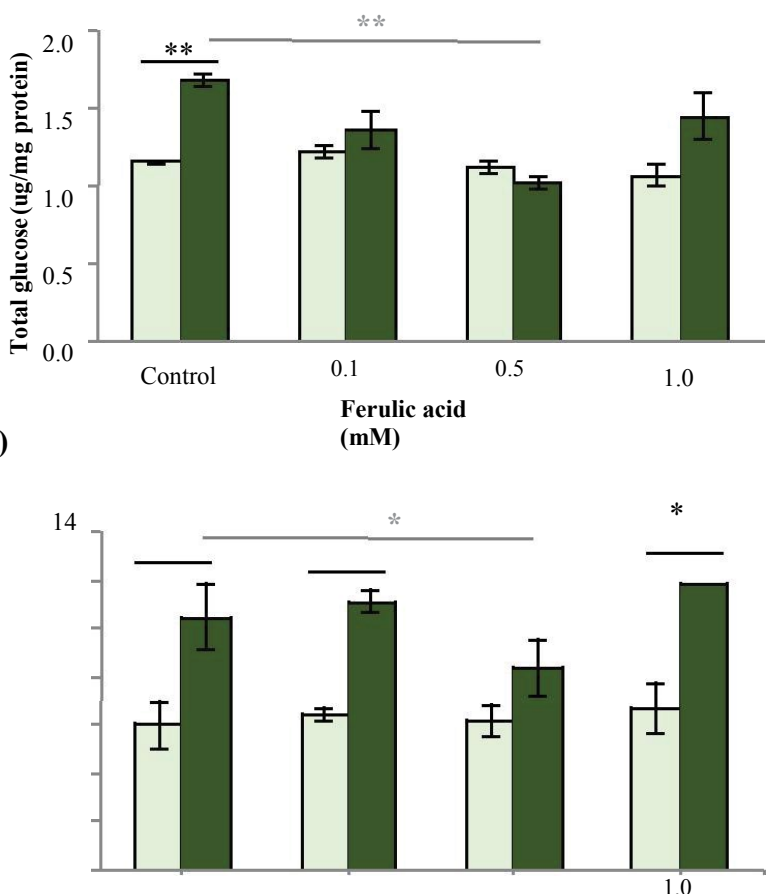

Figure 3: Implications of ferulic acid in energy metabolism of high-sugar fed Drosophila.

Drosophilas were reared on media supplemented with FA. After eclosion, flies remained on the FA-inoculated media for 7 days before being transferred to normal media supplemented with FA (light green bars) or a high sugar diet (HSD) media supplemented with FA (dark green bars). Various aspects of metabolic health were assessed including (a) average weight of 10 flies, (b) circulating levels of glucose normalized to I of hemolymph, (c) circulating levels of trehalose normalized to I of hemolymph, (d) whole-body glucose normalized to $\mathrm{mg}$ of protein, (e) whole body trehalose normalized to mg of protein and (f) whole body triglycerides also normalized to $\mathrm{mg}$ of protein. All values represent $n=5$ independent experiments $+/$ - the standard error. Significance is indicated as * $p<0.05$ or ** $p<0.01$ where black lines indicate difference between treatments groups and grey lines indicate the first significance difference between dose groups for the HSD supplemented with FA.

similarly instigated a drastic rescue of dilp expression in HSD-treated flies at the lowest concentration, $0.1 \mathrm{mM}$ (Figures $4 \mathrm{~b}$ and $4 \mathrm{~d} ; p<0.05$ ). Interestingly, FA also reduced dilp5 expression mostly efficiently at 0.1 and $0.5 \mathrm{mM}$ (Figure $4 \mathrm{f} ; p<0.01$ ) in contrast to the Lf5221 group which did not have a significant effect on dilp5 expression.

\section{FA-producing L. fermentum NCIMB 5221 reduced obesity markers in Drosophila fed a high-fat diet}

Drosophila reared on a media supplemented with various concentrations of Lf5221 were allowed to age for 7 days before being transferred to a high-fat diet (HFD) media containing $20 \%$ coconut oil along with the identified concentrations of Lf5221. After three weeks, the mass of flies on the HFD was significantly elevated by $32 \%$ as compared to controls (Figure 5a; $p<0.01$ ). Flies co-treated with Lf5221, but not heat-inactivated Lf5221, demonstrated a dose-dependent decrease in body mass where at a dosage of $2.5 \times 10^{9} \mathrm{CFU} / \mathrm{ml}$ had a reduction of $35 \%$ (Figure $5 \mathrm{a} ; p<0.05$ ). Total glucose and trehalose levels where significantly increased by $27 \%$ (Figure $5 \mathrm{~b} ; p<0.05$ ) and $48 \%$ (Figure 5 c; $p<0.01$ ), respectively. At $5.0 \times 10^{8} \mathrm{CFU} / \mathrm{ml}$, Lf5221 rescued total glucose and trehalose levels to that of controls $(p>0.05)$ where in both measures, the heat-inactivated Lf5221 has no effect. Whole-body triglycerides were significantly elevated by $50 \%$ in HFD treated flies as expected (Figure $5 \mathrm{~d}$; $p<0.01$ ), and dose-dependently reduced in Lf5221-treated flies reaching the level of controls at $2.5 \times 10^{9} \mathrm{CFU} / \mathrm{ml}$ (Figure $5 \mathrm{~d}$; $p<0.01$ ).

Ferulic acid from L. fermentum NCIMB 5221 reduced markers of obesity in high-fat diet fed Drosophila

Flies reared on media containing various concentrations of FA were allowed to age 7 days before be transferred to the previously described HFD. As expected, there was a $62 \%$ increase in body weight (Figure 


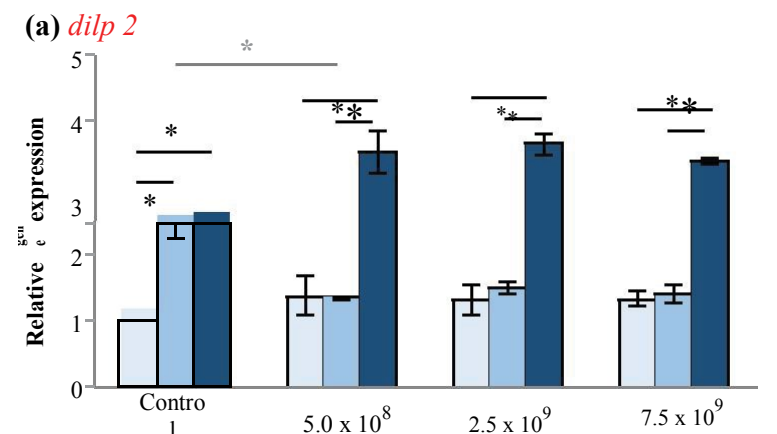

(c) $\operatorname{dilp}$ $5.0 \times 10^{8} \quad 2.5 \times 10$
L. fermentum NCIMB 5221
(CFU/ml)

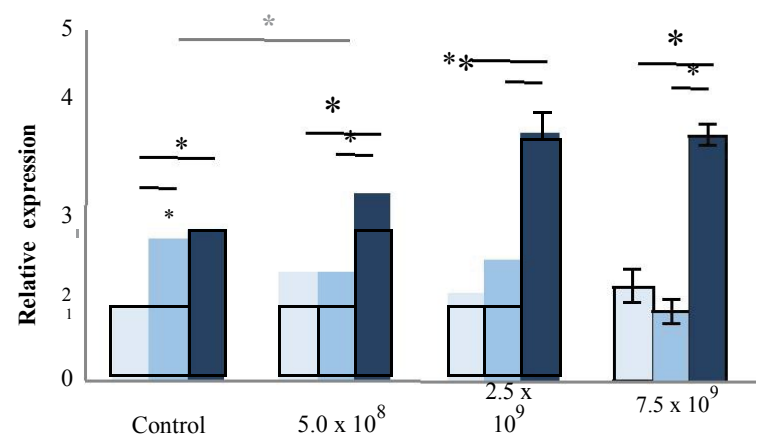

(e) $\operatorname{dilp} 5$

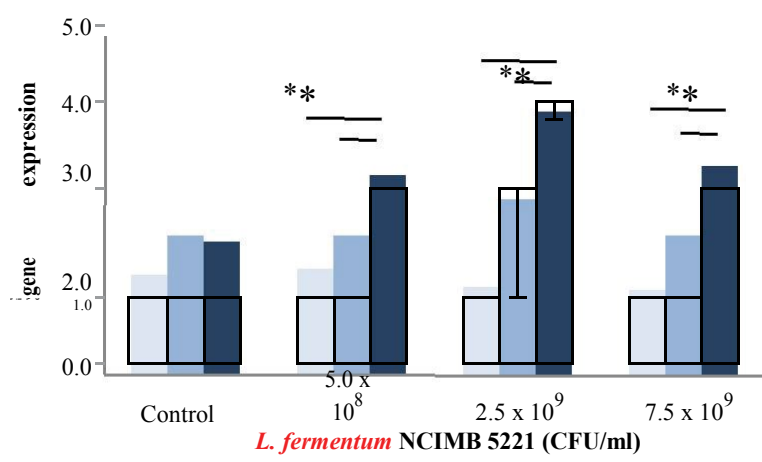

(b) dilp 2

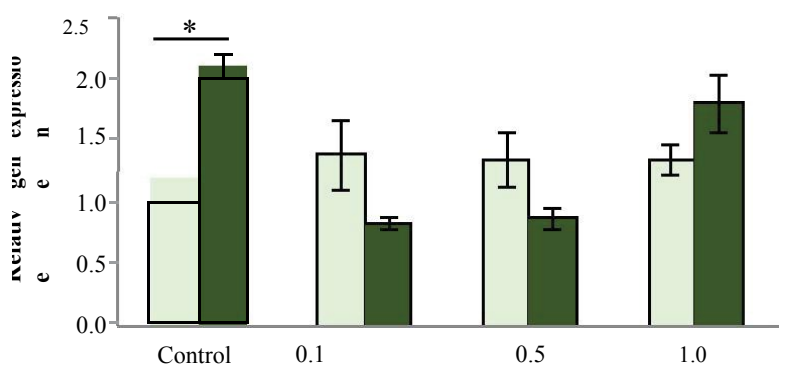

(d) $\operatorname{dilp} 3$

Ferulic acid (mM)

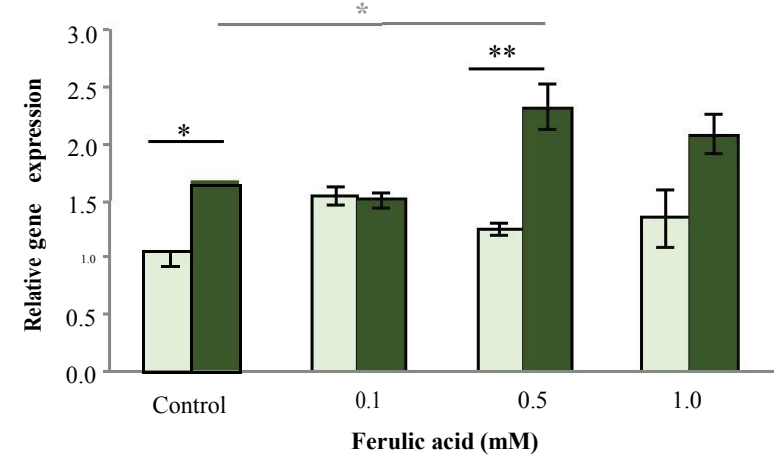

(f) $\operatorname{dilp} 5$

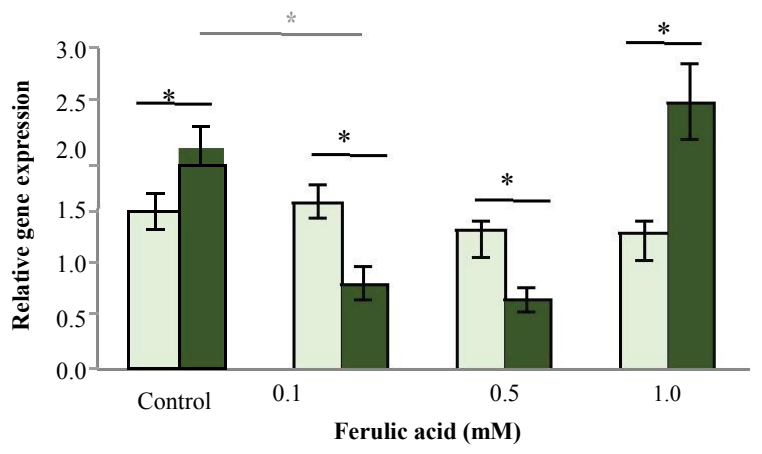

Figure 4: Expression of Drosophila insulin-like peptide (dilp) genes is influenced by L. fermentum NCIMB 5221 and ferulic acid in flies fed a high-sugar diet. Drosophilas were reared on media containing either Lf5221 or FA. After eclosion and 7 days of aging on the treated media, flies were transferred to treated high-sugar diet (HSD) media. Media was treated with either Lf5221 (a,c,e) with control media with live Lf5221 (light-blue bars), HSD with live Lf5221 (medium-blue bars) and HSD with heat-inactivated Lf5221 (dark-blue bars) or FA (b,d,f) with control media supplemented with FA (light green bars) or a HSD supplemented with FA (dark green bars). Expression of (a,b) dilp 2 (c,d) dilp 3 or $(\mathbf{e}, \mathbf{f})$ dilp 5 was assessed with real-time pcr. Each point represents $n=5$ independent replications $+/$ - standard error. Significance is indicated as * $p<0.05$ or ${ }^{* *} p<0.01$ where black lines indicate difference between treatments groups and grey lines indicate the first significance difference between dose groups for the HSD supplemented with Lf5221 or FA.

6a; $p<0.01)$. At a concentration of $0.5 \mathrm{mM}$, FA maximally reduced the weight gain by $25 \%(p<0.01)$. There were also significant increases in both total glucose (Figure 6b) and trehalose (Figure 6c), which were significantly reduced by FA at $0.1 \mathrm{mM}(p<0.01)$ and $0.5 \mathrm{mM}$ $(p<0.05)$, respectively. Finally, there was a significant $60 \%$ increase in whole-body triglycerides that was significantly reduced by $32 \%$ with supplementation of $0.5 \mathrm{mM}$ FA (Figure $6 \mathrm{~d} ; p<0.01$ ).

Fundamental gene expression of genes regulating fatty acid metabolism is altered by ferulic acid produced by $L$. fermentum NCIMB 5221

Acetyl CoA carboxylase (ACC), fatty acid synthase (FAS) and phosphoenolpyruvate carboxykinase (PEPCK) are three enzymes critical for the production of fatty acids (ACC and FAS) and glyceroneogenesis (PEPCK). All three multi-unit enzymes should be significantly elevated in HFD flies reflecting the increase in fatty acid production. ACC was significantly elevated in HFD flies, and reduced with treatment of $5.0 \times 10^{8} \mathrm{CFU} / \mathrm{ml}$ Lf5221 (Figure $7 \mathrm{a} ; p<0.01$ ) but not FA (Figure $7 \mathrm{~b} ; p>0.05$ ). FAS was similarly elevated, yet significantly reduced by both Lf5221 at $5.0 \times 10^{8} \mathrm{CFU} / \mathrm{ml}$ (Figure 7c; $p<0.01$ ) and FA at $0.5 \mathrm{mM}$ (Figure $7 \mathrm{~d} ; p<0.01$ ). Finally, PEPCK was also elevated in HFD-fed Drosophila and significantly reduced by both Lf5221 at $2.5 \times 10^{9} \mathrm{CFU} / \mathrm{ml}$ (Figure $7 \mathrm{e} ; p<0.01$ ) and by FA at $0.5 \mathrm{mM}$ (Figure $7 \mathrm{f}$; $p<0.01)$. 
(a)

(c)

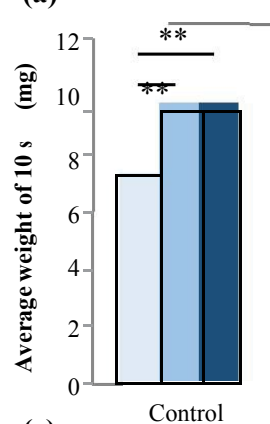

$*$

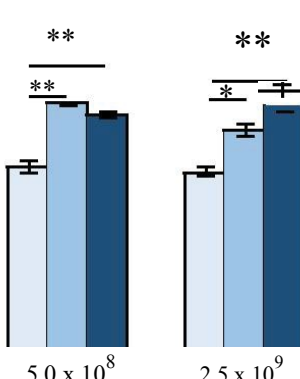

$5.0 \times 10^{8}$

$2.5 \times 10^{9}$

L. fermentum NCIMB 5221 (CFU/ml)

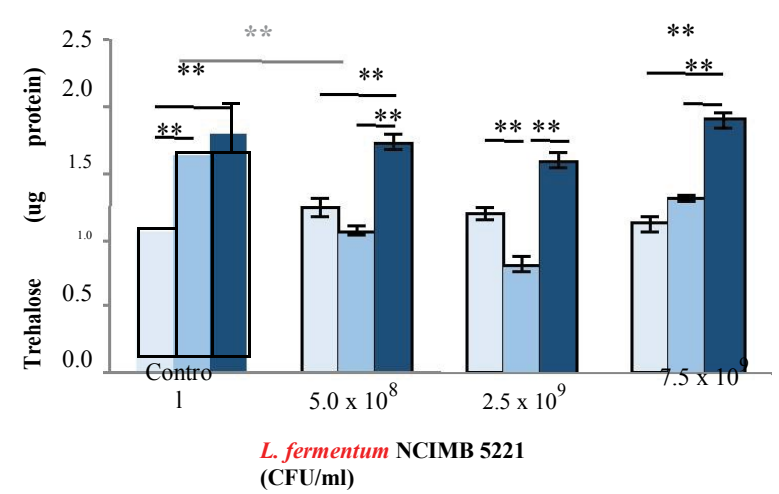

(b)

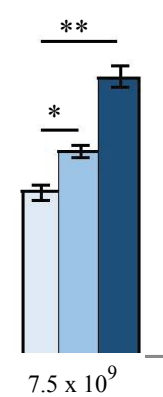

(d)

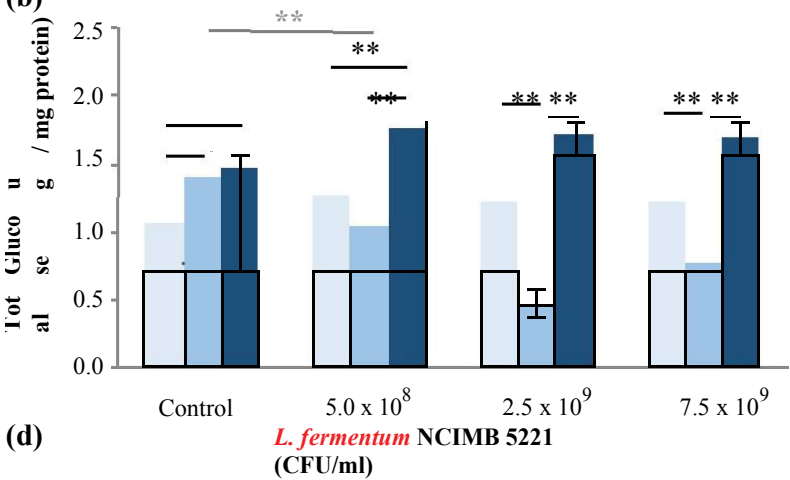

L. fermen

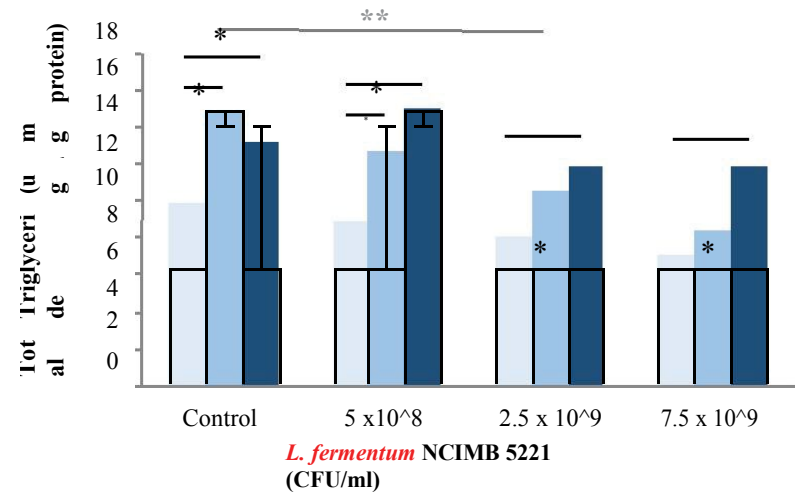

$(\mathrm{CFU} / \mathrm{ml})$

Figure 5: Implications of L. fermentum NCIMB 5221 in energy metabolism of high-fat fed Drosophila.

Drosophilas were reared on media containing various concentrations of Lf5221. After eclosion, flies remained on the Lf5221-inoculated media for 7 days and were then subsequently transferred to the high-fat diet (HFD) media containing Lf5221 for 21 days to induce obesity. All markers were measured in Lf5221 inoculated control (light-blue bars), HFD with live Lf5221 (medium-blue bars) and HFD with heat-inactivated Lf5221 (dark-blue bars)

treated groups at doses of $0,5.0 \times 10^{8}, 2.5 \times 10^{9}$ and $7.5 \times 10^{9} \mathrm{CFU} / \mathrm{ml}$. Various aspects of metabolic health were assessed including (a) average weight of 10 flies, (b) whole-body glucose normalized to $\mathrm{mg}$ of protein, (c) whole body trehalose normalized to $\mathrm{mg}$ of protein and (d) whole body triglycerides also normalized to mg of protein. All values represent $n=5$ independent experiments $+/$ - the standard error. Significance is indicated as * $p<0.05$ or ${ }^{* *} p<0.01$ where black lines indicate difference between treatments groups and grey lines indicate the first significance difference between dose groups for the HFD supplemented with Lf5221.

\section{FA-producing L. fermentum NCIMB 5221 attenuates the fundamental mechanisms of IlS}

To determine if the FA produced from Lf5221 affects the fundamental signaling mechanisms of insulin-like signaling (IIS), the genetic expression of dTOR, dAkt and dFOXO was analyzed by realtime PCR. As expected, HSD reduced the expression of the negative regulator of insulin, dFOXO while elevating dAkt and dTOR (Figure 8). In response to Lf5221, gene expression was rescued for dFOXO at $5.0 \times 10^{8} \mathrm{CFU} / \mathrm{ml}$ (Figure 8a; $p<0.01$ ), reduced for dAkt at $5.0 \times 10^{8}$ $\mathrm{CFU} / \mathrm{ml}$ (Figure $8 \mathrm{c}, p<0.05$ ) and rescued for $\mathrm{dTOR}$ at $5.0 \times 10^{8}$ and 7.5 $\times 10^{9} \mathrm{CFU} / \mathrm{ml}$ (Figure $8 \mathrm{e} ; p>0.05$ ). Similarly for FA, dFOXO expression was rescued at $0.5 \mathrm{mM} \mathrm{FA}$ (Figure $8 \mathrm{~b} ; p>0.05$ ), dAkt was rescued at 1.0 $\mathrm{mM}$ (Figure $8 \mathrm{~d} ; p>0.05$ ) and dTOR was rescued at $0.5 \mathrm{mM}$ (Figure 8f; $p>0.05$ ). This indicates that the rescued effects of HSD on Drosophila from orally-treated Lf5221 and FA is due to its modulatory action on fundamental IlS pathways.

\section{Discussion}

Metabolic syndrome is a collection of diverse metabolic phenotypes including insulin resistance, an increase in abdominal body fat, increased blood pressure, and abnormal cholesterol and triglyceride levels [2]. Current therapies include statins to control cholesterol, ACE inhibitors to lower blood pressure and various anti-diabetic medications. These are all limited approaches to treating this multi-faceted disease as they only target one of the symptoms of metabolic syndrome, do not address the underlying cause and elicit several unwanted side effects. In the current study, the application of a single probiotic bacterium, L. fermentum NCIMB 5221 (Lf5221) towards the reduction of several metabolic syndrome markers was assessed. It was found that through the activity of Lf5221's metabolic product, ferulic acid (FA), markers of both diabetes and obesity were rescued to levels of control both on physiological and molecular levels possibly through the regulation of downsteam IlS signaling factors including dTOR.

Alone, Lf5221 fed to Drosophila melanogaster at the dosage range from $5.0 \times 10^{8}$ to $7.5 \times 10^{9} \mathrm{CFU} / \mathrm{ml}$ elicited a dose-dependent reduction in body weight and total glucose and trehalose levels in the diet-induced diabetic model and a reduction in the body weight, total glucose, trehalose and triglyceride levels in the diet-induced obesity model. In the diabetes model, the diabetic phenotypes were completely rescued at $2.5 \times 10^{9} \mathrm{CFU} / \mathrm{ml}$, which is consistent with a physiological dose in both animals and humans [21-25]. Considering that the effect of Lf5221 on these physiological markers of diabetes and obesity is lost when flies are they are fed on heat-inactivated Lf5221, it can be deduced that a secreted metabolic product of Lf5221 is responsible for the effects and not a signaling cascade implemented through a surface ligand. One of the main secreted products from Lf5221 is FA, produced through its intrinsic ferulic acid esterase (FAE) activity. 
(a)

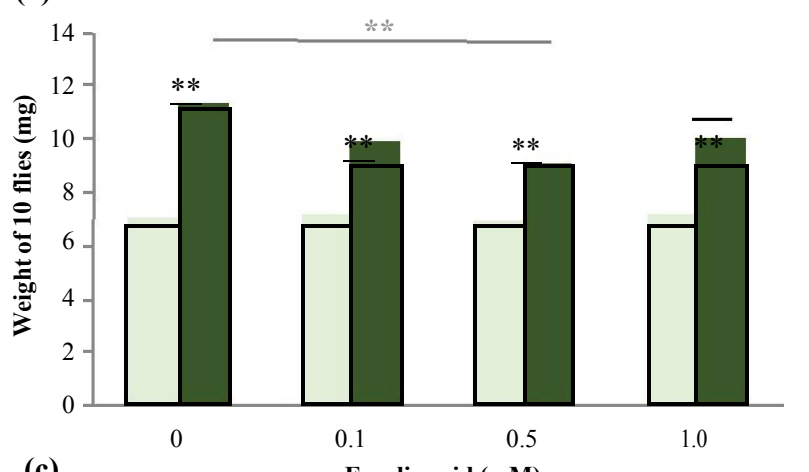

(c)

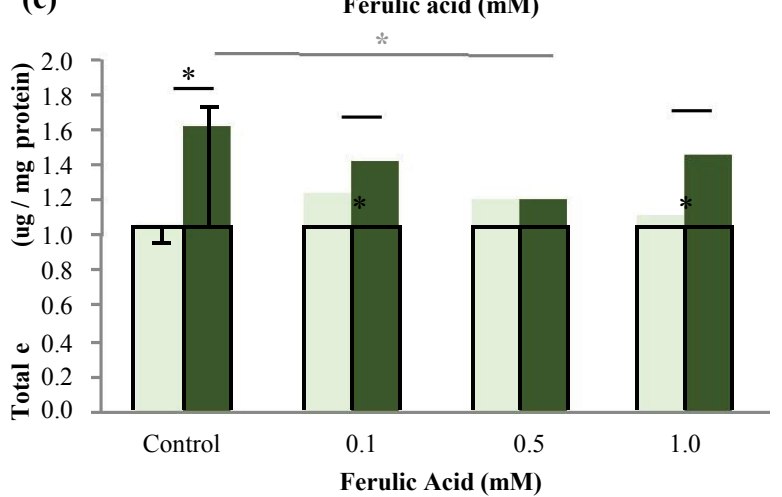

(b)

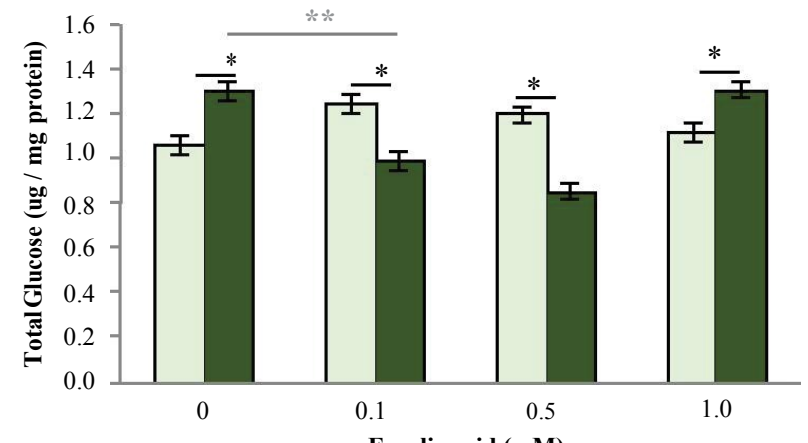

Ferulic acid (mM)

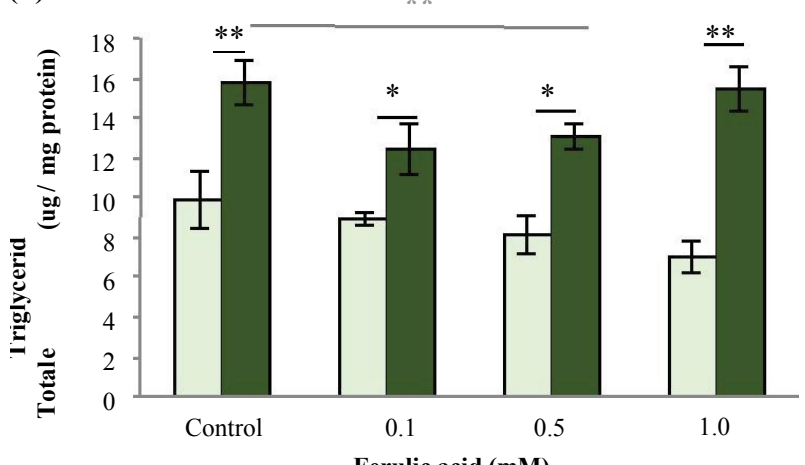

Figure 6: Implications of ferulic acid in energy metabolism of high-fat fed Drosophila.

Flies were reared on media containing various concentrations of ferulic acid. After eclosion, flies remained on the FA-inoculated media for 7 days and were then subsequently transferred to the high-fat diet (HFD) media containing FA for 21 days to induce obesity. All markers were measured in FA treated control (light-green bars), HFD supplemented with FA (dark-green bars). Various aspects of metabolic health were assessed including (a) average weight of 10 flies, (b) whole-body glucose normalized to $\mathrm{mg}$ of protein, (c) whole body trehalose normalized to $\mathrm{mg}$ of protein and (d) whole body triglycerides also normalized to mg of protein All values represent $n=5$ independent experiments $+/$ - the standard error. Significance is indicated as * $p<0.05$ or ${ }^{* *} p<0.01$ where black lines indicate difference between treatments groups and grey lines indicate the first significance difference between dose groups for the HFD supplemented with FA.

Previously, there have been several indications in human clinical trials that probiotics have a beneficial effect on various markers of metabolic syndrome including B. lactis [26], L. plantarum [27], L. rhamnosus [28], L. gasseri [29] and L. reuteri [25]. There are also conflicting studies showing that probiotics have a less beneficial effect on the aforementioned markers including L. casei Shirota [30]. Despite there being several studies indicating a beneficial effect of probiotics on one or more markers of metabolic syndrome, very few studies have addressed the underlying mechanisms of communication between the probiotic species and the host metabolic pathways.

Probiotics can alter the composition of the gut microflora in several ways including i) directly binding to the gut epithelial layer through surface ligands instigating downstream signaling cascades, ii) altering the broad composition of the microflora community in the GIT hence altering the efficiency of energy extraction from foods and mineral absorption and iii) producing bacterial metabolites that activate downstream signaling pathways that have broad implications on host physiology. In this study, the secreted metabolite of Lf5221, FA was specifically investigated in the context of metabolic syndrome to delineate a plausible mechanism of communication by Lf5221 to the metabolic health of the host.

FA is a well-known hydroxycinnamic phenolic phytochemical with anti-inflammatory and anti-oxidative activities [31]. It has also been previously shown that FA has several metabolic benefits. In a high-fat diet induced obesity model in mice, FA at doses of 25 and $50 \mathrm{mg} / \mathrm{kg}$ body weight reduced elevated blood glucose and serum leptin levels, lowered the insulin resistance and increased the serum adiponectin levels, serum lipid levels and liver cholesterol and triglyceride accumulations. Further, this study found that FA reduced the expression of hepatic lipogenic genes such as sterol regulatory element-binding protein $1 \mathrm{c}$ (SREBP1c), fatty acid synthase (FAS) and acetyl-CoA carboxylase (ACC) along with upregulating hepatic carnitine palmitoyltransferase 1a (CPT1a) and peroxisome proliferator activated receptor alpha (PPARa) [32]. FA at a dose of $2.6 \mathrm{mmol} / \mathrm{kg}$ diet was effective at reducing inflammatory, hyperlipidemia, hyperglycemia markers in a highfructose, high-fat diet-induced model of metabolic syndrome [33]. A similar study found that FA minimized insulin resistance, vascular dysfunction and remodeling in a rat model of high-carbohydrate, highfat diet-induced metabolic changes, which may be realized through suppression of oxidative stress, down-regulation of $\mathrm{p} 47 \mathrm{phox}$, increased nitric oxide bioavailability with up-regulation of endothelial nitric oxide synthase and suppression of tumor necrosis factor- $\alpha$ (TNF- $\alpha$ ) [34]. It is clear from these studies that FA simultaneously targets several aspects of metabolic syndrome including hyperglycemic indices, hypercholesterolemia markers, inflammation, oxidation and others through impacting the fundamental signaling mechanisms underlying energy metabolism. This makes FA an ideal candidate to study the effects of metabolic syndrome.

In the present study, the metabolic action of ferulic acid was confirmed. FA at a dose of $0.5 \mathrm{mM}$ rescued the circulating glucose and trehalose as well as whole-body glucose and trehalose in the HSD 
(a)

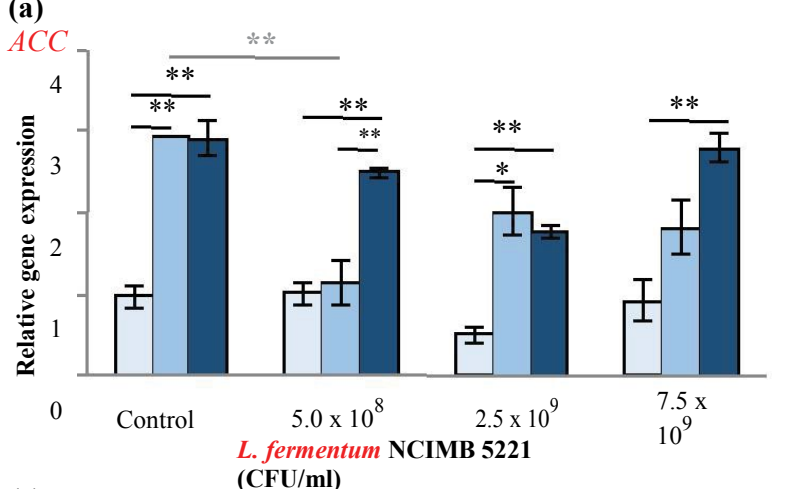

(c)

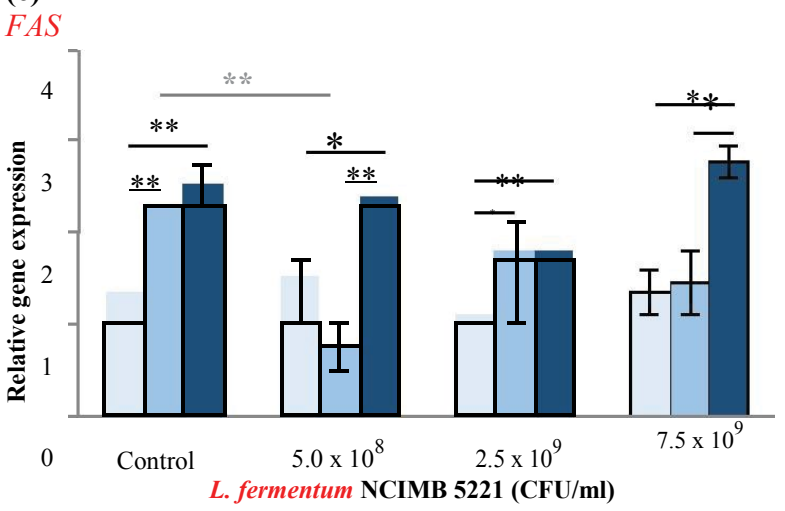

(e) $P E P C K$

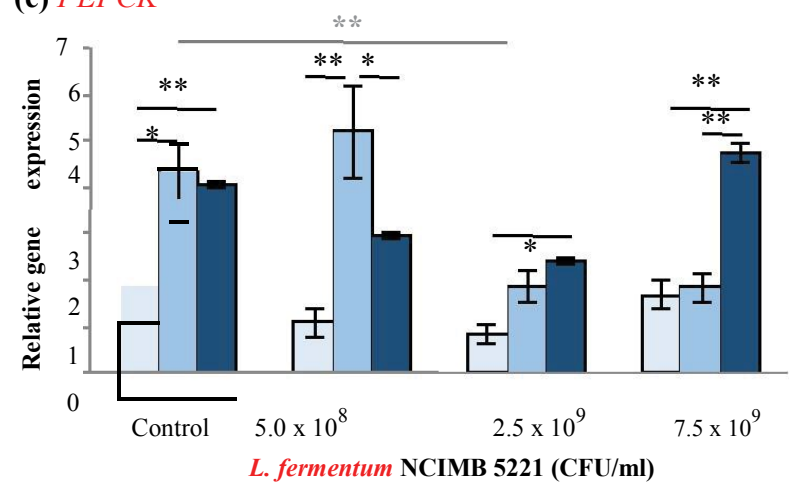

(b) $A C C$

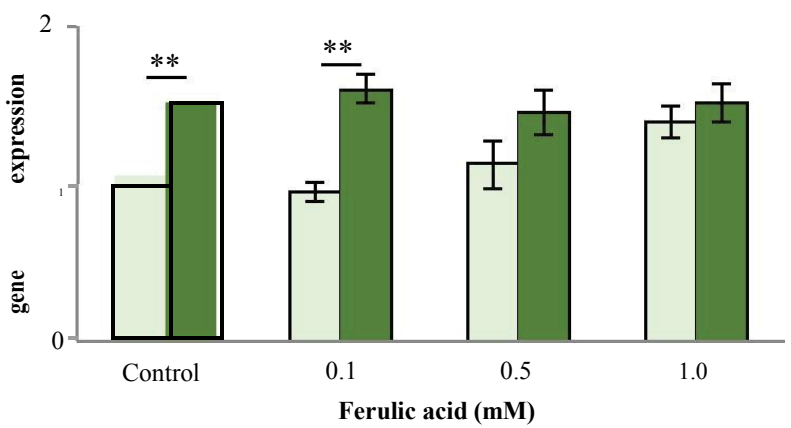

(d) $F A S$

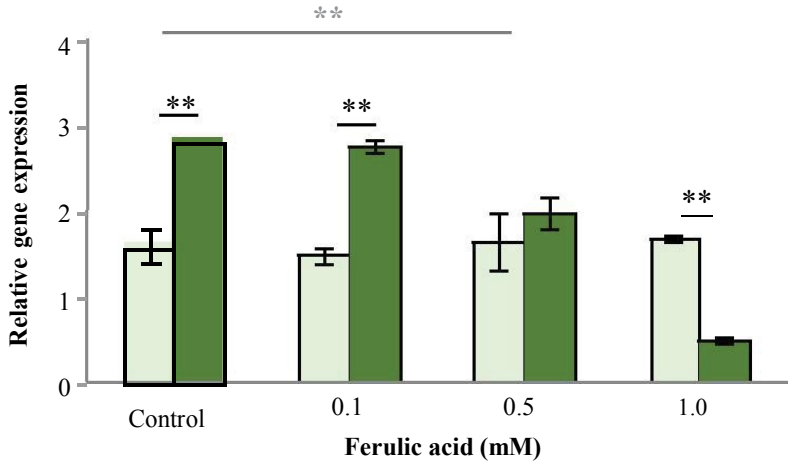

(f)

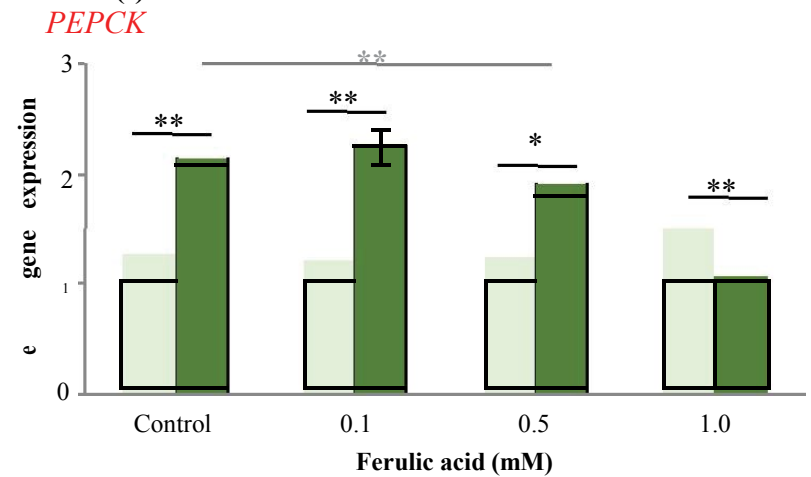

Figure 7: Expression of fatty acid metabolic genes in HFD fed flies exposed to L. fermentum NCIMB 5221 or Feurlic acid.

Drosophilas were reared on media containing either Lf5221 or FA. After eclosion and 7 days of aging on the treated media, flies were transferred to treated high-fat diet (HFD) media. Media was treated with either Lf5221 (a,c,e) with control media with live Lf5221 (light-blue bars), HFD with live Lf5221 (medium-blue bars) and HFD with heat-inactivated Lf5221 (dark-blue bars) or FA (b,d,f) with control media supplemented with FA (light green bars) or a HFD supplemented with FA (dark green bars). Expression of (a,b) $A C C$ (c,d) FAS or (e,f) PEPCK was assessed with real-time pcr. Each point represents $n=5$ independent replications $+/-$ standard error. Significance is indicated as ${ }^{*} p<0.05$ or ${ }^{* *} p<0.01$ where black lines indicate difference between treatments groups and grey lines indicate the first significance difference between dose groups for the HFD supplemented with FA.

fed flies. In the HFD flies, FA at a dose of $0.5 \mathrm{mM}$ rescued the total glucose, trehalose and significantly reduced the total triglyceride levels as compared to untreated groups. Especially considering the effects on the circulating levels of glucose and trehalose, the isolated FA treatment seemed to be more effective than Lf5221. This could be due to the higher concentrations of ferulic acid reaching the various organ systems in the fly.

In mammals and flies alike, insulin resistance is determined through the overproduction of insulin and insulin-like growth factor signaling in response to circulating levels of glucose. In mammals, it is the expression of insulin-like receptor and glucagon-like peptide that are altered when insulin resistance is developed. In flies, it is the production of the 8 Drosophila insulin-like peptides (dilps) and the Drosophila insulin receptor (DInR). It was found that signaling and activation of the DInR through tyrosine phosphorylation stimulates dilp 2 or dilp 5 in the insulin-producing cells in the Drosophila neural net [35]. Further, altered expression of the genes encoding dilp 2, 3, 5 and 6 results in modulated IlS and profound metabolic and longevity consequences [36]. In addition, nutrient status will activate dilp expression through the activation of Unpaired 2 (Upd2). The function of each of the dilp homologs has not been fully elucidated but it has been postulated that dilp 2 is involved in carbohydrate and trehalose 


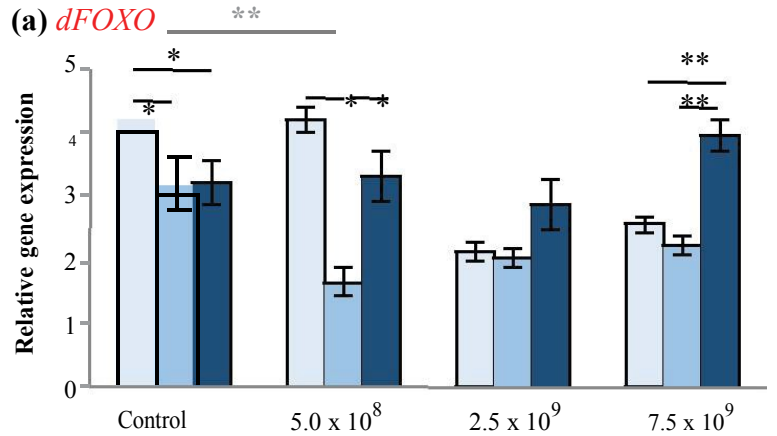

(c) $d A k t$

L. fermentum NCIMB 5221 (CFU/ml)

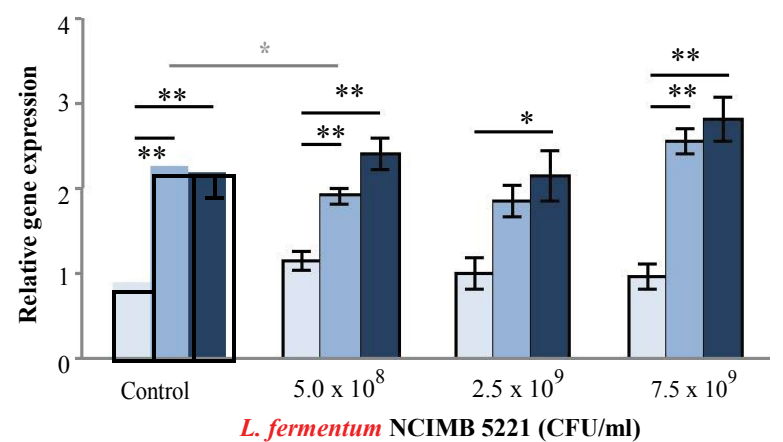

(e) $d T O R$

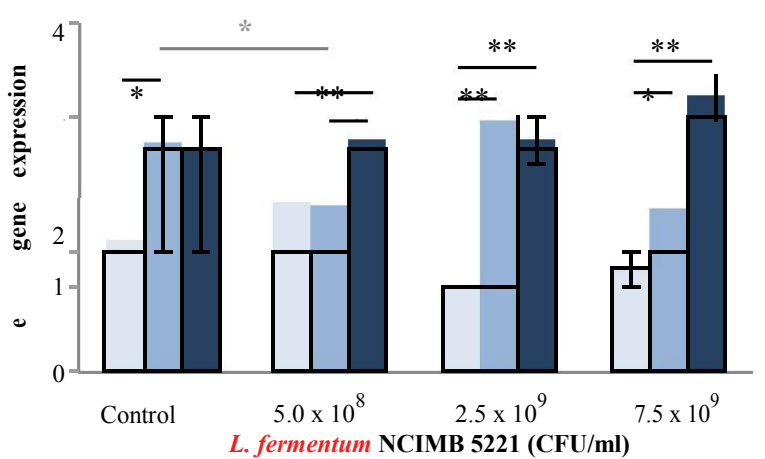

(b) $d F O X O$

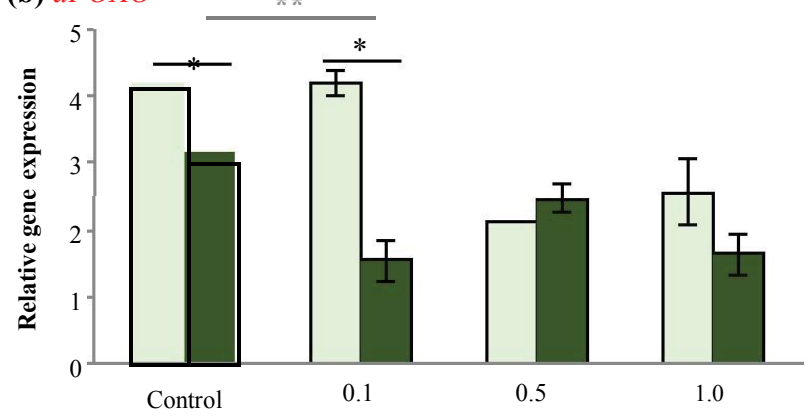

(d) $d A k t$

Ferulic acid (mM)

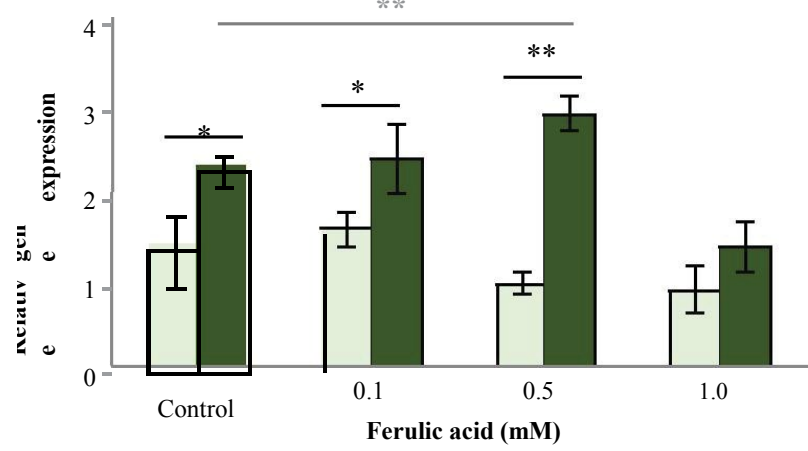

(f) $d T O R$

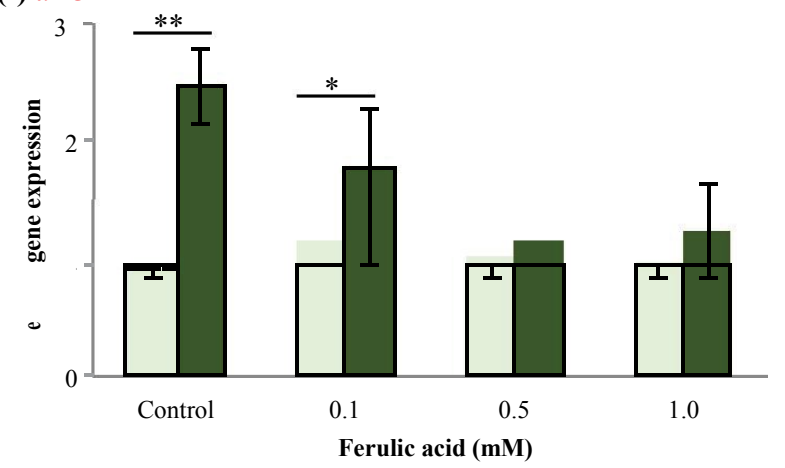

Figure 8: Expression of upstream regulators of insulin signaling in Drosophila melanogaster fed a HSD with L. fermentum NCIMB 5221 or ferulic acid. Drosophilas were reared on media containing either Lf5221 or FA. After eclosion and 7 days of aging on the treated media, flies were transferred to treated highsugar diet (HSD) media. Media was treated with either Lf5221 (a,c,e) with control media with live Lf5221 (light-blue bars), HSD with live Lf5221 (medium-blue bars) and HSD with heat-inactivated Lf5221 (dark-blue bars) or FA (b,d,f) with control media supplemented with FA (light green bars) or a HSD supplemented with FA (dark green bars). Expression of (a,b) $d F O X O(\mathbf{c}, \mathbf{d}) d A k t$ or $(\mathbf{e}, \mathbf{f}) d T O R$ was assessed with real-time pcr. Each point represents $n=5$ independent replications $+/-$ standard error. Significance is indicated as ${ }^{*} p<0.05$ or ${ }^{* *} p<0.01$ where black lines indicate difference between treatments groups and grey lines indicate the first significance difference between dose groups for the HSD supplemented with Lf5221 or FA.

metabolism, dilp 3 in lipid metabolism and dilp 5 in dietary restriction response and longevity [36].

As expected, there was a significant increase in dilp 2, 3 and 5 expression in flies fed a HSD. Lf5221, but not heat-inactivated Lf5221, efficiently reduced dilp 2 and 3 expression to the level of controls. Similar to the physiological results, FA was more efficient at reducing dilp 2, 3 and 5 expression levels at both $0.1 \mathrm{mM}$ and $0.5 \mathrm{mM}$ doses. This confirms that it is the metabolic activity of Lf5221 that is eliciting the gene-dependent regulation of dilps and not passive signaling, possibly through the action of FA. As previously outlined, it is not known at what level the gut microbiota, and its metabolites are influencing the regulation of insulin signaling. The downstream IlS signaling factors are the same in Drosophila and mammals so the expression of three of the key mediators, dFOXO, dAkt and dTOR, was assessed.

In mammals and flies alike, Akt and TOR are induced downstream of insulin signaling, which subsequently suppresses FOXO [37]. Likewise, in flies fed on a HSD, there was a significant elevation in dAkt and dTOR, and a decrease in dFOXO. Notably, dFOXO and dTOR, but not dAkt expression was rescued at $2.5 \times 10^{9} \mathrm{CFU} / \mathrm{ml}$ Lf5221 treatment. Similarly, FA treatment at $0.5 \mathrm{mM}$ rescued dTOR and dFOXO at 0.5 $\mathrm{mM}$ and dAkt at $1.0 \mathrm{mM}$. Notably, the downstream signaling factors dTOR and dFOXO were affected by Lf5221 treatment, but not dAkt indicating that the regulation of insulin signal in flies is controlled downstream of dAkt. 
This result is significant as dTOR is the key energy-sensing molecule in the body acting as an integrator of intrinsic molecular and hormonal cues with environmental nutritional signals. It is well known that the mammalian mTORC is dysregulated in patients with diabetes, obesity and metabolic syndrome [38]. Through IlS pathways, TOR signaling (via the complex mTORC1 in mammals) is induced by the phosphorylation and inactivation of the upstream inhibitors of TOR, TSC1/2, by Akt. Hence, through Rheb signaling, TOR is disinhibited and allowed to bind to its effector co-factors. In contrast, mTORC1 is also inhibited by AMP-activated protein kinase (AMPK), an energysensing molecule that is activated in response to fluctuations in the AMP:ATP ratios. AMPK integrates several energy-regulating cues including glucose uptake, $\beta$-oxidation of fatty acids and the biogenesis of glucose transporters. Nutritionally, mTORC is directly regulated by high levels of free amino acids and growth factors including IGF-1/2 [39]. Interestingly, one study has indicated that the growth-promoting properties of a commensal gut microbiota L. plantarum in Drosophila, may act via a dTOR-dependent mechanism, though the method of communication between the gut microbiota and TOR signaling was not elucidated [40].

Interestingly, there have been some indications that ferulic acid can directly impact TOR signaling. For example, one study in HeLa cells showed that ferulic acid inactivated TOR signaling to a similar extent as the well-known inhibitor rapamycin [41]. In contrast, another study in a rodent ischemia model indicated that FA not only had a reduced infarct volume, but also reduced the injury-induced decreases in dTOR and its downstream effectors p70S6 kinase and S6 phosphorylation [42]. In addition, the anti-oxidant and anti-inflammatory actions of FA may also indirectly impact dTOR regulation, which is complex and involves several parallel factors. Nevertheless, the current study strongly suggests that the energy-regulating properties of Lf5221 occur through FA-mediated modulation of dTOR signaling.

One consideration for this study is the bioavailability of FA and the differences in its metabolism between flies and humans. FA is readily and rapidly metabolized by the gut microflora to generate simpler absorbable phenolic compounds [43] including dihydroferulic acid, vanillic acid, protocatechuic acid, p-coumaric acid and caffeic acid [44]. Indeed, it is the activity of these smaller polyphenols that are believed to elicit the anti-oxidant, anti-inflammatory and metabolic effects on the host [45]. However, it could alternatively be the bacterial metabolites secreted from the community of microbes in an environment enriched with the polyphenol elements that elicits the physiological effects. Regardless, the signaling pathways that are affected by the consumption of FA are dependent on the gut microbiota community composition, which varies from person to person. It is also dependent on the lability of the consumed polyphenol by transiting through the entire GIT subjected to the harsh acidity of the stomach and bile secretion in the small intestine. FA delivered orally is absorbed in the upper GI tract and rapidly excreted, providing only a short residence time $[46,47]$ and bioavailability of FA is thus determined by its bioaccessibility [48] (e.g. bran bound FA is more bioavailable than free FA [49]. It is noted that free FA formulations are not available and attempts have been made to use FA rich food, modify FA and process food with very limited successes $[47,50,51]$. The use of a probiotic bacterium that produces FA continuously in the gut could potentially overcome this limitation while providing the synergistic benefits of a probiotic bacterium.

Altogether, consumption of an intrinsically FA-producing probiotic bacterium circumvents both of these issues by secreting FA locally in the GIT thus increasing the active concentration where it is most needed. Also, probiotic bacteria, especially was encapsulated in microcapsules such as the alginate-poly-l-lysine-alginate capsules, has an increases bioavailability in the lower colonic regions where most of the beneficial bacteria that conduct the biotransformation of polyphenolic compounds are found [13].

\section{Conclusion}

Metabolically active probiotic bacteria are a viable option for the treatment of metabolic syndrome as they can simultaneously treat many if not all of the physiological manifestations of metabolic syndrome including hypercholesterolemia, hyperglycemia, insulin resistance, high blood pressure etc. In particular, the commensal FAE-active probiotic bacteria Lf5221 has been shown to be a potent producer of FA. The anti-inflammatory, anti-oxidative and metabolic activity of FA makes it a good therapeutic alternative to the treatment of metabolic syndrome. By supplementing ones diet with an environmentally resistant probiotic bacterium, the active component FA will be released in the lower colonic compartments where its activity is most beneficial circumventing its pending degradation in the harsh environments of the GIT. Further, the action of FA was shown to impact not only the physiological aspects of metabolic syndrome, but also the fundamental IlS cascades, possibly through the modulation of dTOR, an integrator of intrinsic energy regulators and environmental influences. This is highly significant as dTOR is intregral to several energy-regulating diseases including diabetes, obesity and even neurodegeneration. This makes Lf5221 a promising therapeutic agent for not only metabolic syndrome, but any energy-regulating disorder.

\section{References}

1. Burokas A, Moloney RD, Dinan TG, Cryan JF3 (2015) Microbiota regulation of the Mammalian gut-brain axis. Adv Appl Microbiol 91: 1-62.

2. Grundy D (2011) The metabolic syndrome. Atlas of atherosclerosis and metabolic syndrome. 1-26.

3. Aguilar M, Bhuket T, Torres S, Liu B, Wong RJ2 (2015) Prevalence of the metabolic syndrome in the United States, 2003-2012. JAMA 313: 1973-1974.

4. Rochlani Y, Pothineni NV, Mehta JL (2015) Metabolic syndrome: Does it differ between women and men? Cardiovasc Drugs Ther 29: 329-338.

5. Grundy SM (2008) Metabolic syndrome pandemic. Arterioscler Thromb Vasc Biol 28: 629-636.

6. Fukuda S, Ohno H (2014) Gut microbiome and metabolic diseases. Semin Immunopathol 36: 103-114.

7. Power SE, O'Toole PW, Stanton C, Ross RP, Fitzgerald GF (2014) Intestinal microbiota, diet and health. Br J Nutr 111: 387-402.

8. Cani PD, Amar J, Iglesias MA, Poggi M, Knauf C, et al. (2007) Metabolic endotoxemia initiates obesity and insulin resistance. Diabetes 56: 1761-1772.

9. Bäckhed F, Ding H, Wang T, Hooper LV, Koh GY, et al. (2004) The gut microbiota as an environmental factor that regulates fat storage. Proc Natl Acad Sci U S A 101: 15718-15723.

10. Ley RE, Turnbaugh PJ, Klein S, Gordon JI (2006) Microbial ecology: human gut microbes associated with obesity. Nature 444: 1022-1023.

11. Ridaura VK, Faith JJ, Rey FE, Cheng J, Duncan AE, et al. (2013) Gut microbiota from twins discordant for obesity modulate metabolism in mice. Science 341 1241214.

12. Smits HH, Engering A, Kleij D, Jong EC, Schipper K et al. (2005) Selective probiotic bacteria induce IL-10-producing regulatory $T$ cells in vitro by modulating dendritic cell function through dendritic cell-specific intercellular adhesion molecule 3-grabbing non-integrin. J Allergy Clin Immunol 115: 1260-1267.

13. Tomaro-Duchesneau C, Saha S, Malhotra M, Coussa-Charley M, Kahouli I, et al. (2012) Probiotic ferulic acid esterase active Lactobacillus fermentum NCIMB 5221 APA microcapsules for oral delivery: Preparation and in vitro characterization. Pharmaceuticals 5: 236-248.

14. Rachmilewitz D, Katakura K, Karmeli F, Hayashi T, Reinus C, et al. (2004) Toll- 
like receptor 9 signaling mediates the anti-inflammatory effects of probiotics in murine experimental colitis. Gastroenterology 126: 520-528.

15. Kumar M, Nagpal R, Kumar R, Hemalatha R, Verma V, et al. (2012) Cholesterollowering probiotics as potential biotherapeutics for metabolic diseases. Experimental Diabetes Research 2012: 902917.

16. Luoto TM, Haikonen S, Haapasalo H, Goebeler S, Huhtala H, et al. (2009) Large vessel cerebral atherosclerosis is not in direct association with neuropathological lesions of Alzheimer's disease. Eur Neurol 62: 93-98.

17. Scott KP, Antoine JM, Midtvedt T, van Hemert S4 (2015) Manipulating the gut microbiota to maintain health and treat disease. Microb Ecol Health Dis 26 25877

18. Westfall, Lomis N, Singh SP, Prakash S (2015) The gut microflora and its metabolites regulate the molecular cross-talk between diabetes and neurodegeneration. J Diabetes Metab 6: 1-16.

19. de Vries RP, Michelsen B, Poulsen CH, Kroon PA, Heuvel RH, et al. (2011) The faeA genes from Aspergillus niger and Aspergillus tubingensis encode ferulic acid esterases involved in degradation of complex cell wall polysaccharides. Applied and Environmental Microbiology. 63: 4638-4644.

20. Mancuso C, Santangelo R2 (2014) Ferulic acid: pharmacological and toxicological aspects. Food Chem Toxicol 65: 185-195.

21. Luo J, Lushchak OV, Goergen P, Williams MJ, Nassel DR (2014) Drosophila insulin-producing cells are differentially modulated by serotonin and octopamine receptors and affect social behavior. PLoS ONE 9: e99732.

22. Okamura T, Shimizu H, Nagao T, Ueda R, Ishii S (2007) ATF-2 regulates fat metabolism in Drosophila. Mol Biol Cell 18: 1519-1529.

23. Ha EM, Lee KA, Seo YY, Kim SH, Lim JH, et al. (2009) Coordination of multiple dual oxidase-regulatory pathways in responses to commensal and infectious microbes in drosophila gut. Nat Immunol 10: 949-957.

24. Jones ML, Martoni CJ, Di Pietro E, Simon RR, Prakash S (2012) Evaluation of clinical safety and tolerance of a Lactobacillus reuteri NCIMB 30242 supplement capsule: a randomized control trial. Regul Toxicol Pharmacol 63: 313-320.

25. Jones ML, Martoni CJ, Parent M, Prakash S (2012) Cholesterol-lowering efficacy of a microencapsulated bile salt hydrolase-active Lactobacillus reuter NCIMB 30242 yoghurt formulation in hypercholesterolaemic adults. $\mathrm{Br} \mathrm{J}$ Nutr 107: 1505-1513.

26. Bernini LJ, Simao ANC, Alfieri DF, Lozovoy MAB, Mari NL, et al. (2016) Beneficial effects of Bifidobacterium lactis on lipid profile and cytokines in patients with metabolic syndrome: A randomized trial. Effects of probiotics on metabolic syndrome. Nutrition 32: 716-719.

27. Barreto FM, Colado AN, Morimoto HK, Batisti MA, Dichi I, et al. (2014) Beneficia effects of Lactobacillus plantarum on glycemia and homocysteine levels in postmenopausal women with metabolic syndrome. Nutrition 30: 939-942.

28. Sanchez M, Darimont C, Drapeau V, Emady-Azar S, Lepage M5, et al. (2014) Effect of Lactobacillus rhamnosus CGMCC1.3724 supplementation on weight loss and maintenance in obese men and women. Br J Nutr 111: 1507-1519.

29. Kadooka Y, Sato M, Imaizumi K, Ogawa A, Ikuyama K, et al. (2010) Regulation of abdominal adiposity by probiotics (Lactobacillus gasseri SBT2055) in adults with obese tendencies in a randomized controlled trial. Eur J Clin Nutr 64: 636643

30. Tripolt NJ, Leber B, Triebl A, Kofeler H, Stadlbauer V (2015) Effect of Lactobacillus casei Shirota supplementation on trimethylamine- $\mathrm{N}$-oxide levels in patients with metabolic syndrome: An open-label, randomized study. Atherosclerosis 242: 141-144.

31. Alam MA, Subhan N, Hossain H, Hossain M, Reza HM, et al. (2016) Hydroxycinnamic acid derivatives: a potential class of natural compounds for the management of lipid metabolism and obesity. Nutrition and Metabolism 13: 27.

32. Naowaboot J, Piyabhan P, Munkong N, Parklak W, Pannangpetch P (2016) Ferulic acid improves lipid and glucose homeostasis in high-fat diet-induced obese mice. Clin Exp Pharmacol Physiol 43: 242-250.

33. Wang O, Liu J, Cheng Q, Guo X, Wang Y, et al. (2015) Effects of ferulic acid and gamma-oryzanol on high-fat and high-fructose diet-induced metabolic syndrome in rats. PLoS ONE 10: e0118135.

34. Senaphan K, Kukongviriyapan U, Sangartit W, Pakdeechote P, Pannangpetch $P$, et al. (2015) Ferulic acid alleviates changes in a rat model of metabolic syndrome induced by high-Carbohydrate, high-fat diet. Nutrients 7: 6446-6464.
35. Rulifson EJ, Kim SK, Nusse R (2002) Ablation of insulin-producing neurons in flies: growth and diabetic phenotypes. Science 296: 1118-1120.

36. Kannan K, Fridell YW (2013) Functional implications of Drosophila insulin-like peptides in metabolism, aging and dietary restriction. Front Physiol 4: 288

37. Krebs M, Brunmair B, Brehm A, Artwohl M, Szendroedi J, et al. (2007) The Mammalian target of rapamycin pathway regulates nutrient-sensitive glucose uptake in man. Diabetes 56: 1600-1607.

38. Guo S (2014) Insulin signaling, resistance, and the metabolic syndrome: insights from mouse models into disease mechanisms. J Endocrinol 220: T1 $1 \mathrm{~T} 23$.

39. Perluigi M, Di Domenico F, Butterfield DA (2015) mTOR signaling in aging and neurodegeneration: At the crossroad between metabolism dysfunction and impairment of autophagy. Neurobiol Dis 84: 39-49.

40. Storelli G, Defaye A, Erkosar B, Hols P, Royet J, et al. (2011) Lactobacillus plantarum promotes drosophila systemic growth by modulating hormona signals through TOR-dependent nutrient sensing. Cell Metabolism. Elsevier Inc 14: 403-414.

41. Bian Z, Furuya N, Zheng DM, Oliva Trejo JA, Tada N, et al. (2013) Ferulic acid induces mammalian target of rapamycin inactivation in cultured mammalian cells. Biol Pharm Bull 36: 120-124.

42. Koh $P$ (2013) Ferulic acid attenuates focal cerebral ischemia-induced decrease in p70S6 kinase and S6 phosphorylation. Neuroscience Letters 555: 7-11.

43. Olthof MR, Hollman PC, Katan MB (2001) Chlorogenic acid and caffeic acid are absorbed in humans. J Nutr 131: 66-71.

44. Sadeghi Ekbatan S, Sleno L, Sabally K, Khairallah J, Azadi B, et al. (2016) Biotransformation of polyphenols in a dynamic multistage gastrointestinal model. Food Chem 204: 453-462.

45. Richelle M, Tavazzi I, Offord E (2001) Comparison of the antioxidant activity of commonly consumed polyphenolic beverages (coffee, cocoa, and tea) prepared per cup serving. J Agric Food Chem 49: 3438-3442.

46. Zhao Z, Egashira Y, Sanada H (2004) Ferulic acid is quickly absorbed from rat stomach as the free form and then conjugated mainly in liver. J Nutr 134 3083-3088.

47. Pan Y, Cai L, He S, Zhang Z (2014) Pharmacokinetics study of ferulic acid in rats after oral administration of gamma-oryzanol under combined use of Tween 80 by LC/MS/MS. Eur Rev Med Pharmacol Sci 18: 143-150.

48. Anson NM, Berg R, Havenaar R, Bast A, Haenen G (2009) Bioavailability of ferulic acid is determined by its bioaccessibility. Journal of Cereal Science 49 : 296-300.

49. Rondini L, Peyrat-Maillard MN, Marsset-Baglieri A, Fromentin G, Durand P, et al. (2004) Bound ferulic acid from bran is more bioavailable than the free compound in rat. J Agric Food Chem 52: 4338-4343.

50. Mateo N, Aura M, Selinheimo E, Mattila I, Poutanen K, et al. (2011) Bioprocessing of wheat bran in whole wheat bread increases the bioavailability of phenolic acids in men and exerts anti-inflammatory effects ex vivo. J Nutr 141: 137-143

51. Anson NM, Selinheimo E, Havenaar R, Aura A, Mattila I, et al. (2009) Bioprocessing of wheat bran improves in vitro bioaccessibility and colonic metabolism of phenolic compounds. J Agric Food Chem 57: 6148-6155. 